\title{
Hybrid Adaptive Pinning Control for Function Projective Synchronization of Delayed Neural Networks with Mixed Uncertain Couplings
}

\author{
T. Botmart, ${ }^{1}$ N. Yotha, ${ }^{2}$ P. Niamsup, ${ }^{3}$ and W. Weera ${ }^{4}$ \\ ${ }^{1}$ Department of Mathematics, Khon Kaen University, Khon Kaen 40002, Thailand \\ ${ }^{2}$ Department of Applied Mathematics and Statistics, Rajamangala University of Technology Isan, Nakhon Ratchasima 30000, Thailand \\ ${ }^{3}$ Department of Mathematics, Faculty of Science, Chiang Mai University, Chiang Mai 50200, Thailand \\ ${ }^{4}$ Department of Mathematics, University of Phayao, Phayao 56000, Thailand
}

Correspondence should be addressed to N. Yotha; narongsak.yo@rmuti.ac.th

Received 25 March 2017; Revised 14 June 2017; Accepted 2 July 2017; Published 8 August 2017

Academic Editor: Fathalla A. Rihan

Copyright (C) 2017 T. Botmart et al. This is an open access article distributed under the Creative Commons Attribution License, which permits unrestricted use, distribution, and reproduction in any medium, provided the original work is properly cited.

\begin{abstract}
This paper presents the function projective synchronization problem of neural networks with mixed time-varying delays and uncertainties asymmetric coupling. The function projective synchronization of this model via hybrid adaptive pinning controls and hybrid adaptive controls, composed of nonlinear and adaptive linear feedback control, is further investigated in this study. Based on Lyapunov stability theory combined with the method of the adaptive control and pinning control, some novel and simple sufficient conditions are derived for the function projective synchronization problem of neural networks with mixed time-varying delays and uncertainties asymmetric coupling, and the derived results are less conservative. Particularly, the control method focuses on how to determine a set of pinned nodes with fixed coupling matrices and strength values and randomly select pinning nodes. Based on adaptive control technique, the parameter update law, and the technique of dealing with some integral terms, the control may be used to manipulate the scaling functions such that the drive system and response systems could be synchronized up to the desired scaling function. Finally, numerical examples are given to illustrate the effectiveness of the proposed theoretical results.
\end{abstract}

\section{Introduction}

Presently, neural networks are under extensive consideration because of their significant application in various fields such as image processing, pattern recognition, and associative memories because the switching speed of information processing and the inherent neuron communication is limited [ 1 , 2]. Global asymptotic stability of a general class of recurrent neural networks with time-varying delays is discussed in [3]. Zhang and Han [4] investigated the global asymptotical stability analysis for delayed neural networks using a matrixbased quadratic convex approach. It is well know that the time delay is continually necessarily existent in neural networks because it might lead to inconstancy or considerably inferior performances. So, the neural networks with time delays have attracted considerable attention of many researchers [5-7].
In addition, much attention has been paid to the potential applications of the synchronization of coupled neural networks, for example, secure communication [8-10]. The synchronization criteria for coupled stochastic neural networks with time-varying delays and leakage delay were discussed in [11]. Chen and Cao [12] suggested projective synchronization of neural networks with mixed time-varying delays and parameter mismatch. Moreover, there is synchronization problem that is called function projective synchronization that has received increasing attention in [13-15]. FPS is the driver and response system that can be synchronized up to a scaling function. Many researches mentioned that function projective synchronization (FPS) is the greater general definition of chaotic synchronization [16-18]. It is obvious that the definition of FPS includes comprehensive synchronization and projective synchronization. In order for scaling function 
to achieve unity or be constant, only one complete synchronization or projective synchronization could be obtained since the unpredictability of the scaling function in FPS can also raise the security of communication. Thus, FPS has drawn the attention of many researchers in various fields. In Gao et al. [19] the generalized function projective synchronization of weighted cellular neural networks with multiple time-varying coupling delays was studied. Adaptive projective synchronization in complex networks with timevarying coupling delay discussed in [20]. Tang and Wong [21] studied on the distributed synchronization problem of coupled neural networks by randomly occurring control method. Hu et al. [22] suggested a pinning synchronization control scheme for a class of linearly coupled neural networks. Huang et al. [23] investigated the stabilization of delayed chaotic neural networks by periodically intermittent control. Further, Cai et al. [24] considered the outer synchronization between two hybrid-coupled delayed dynamical networks via aperiodically adaptive intermittent pinning control. However, not all of the neural networks could synchronize by themselves. So, they need to bring the suitable controllers in order to make them synchronize. One of the most important existing control methods is the pinning control.

Pinning control is the strategy that employs the local feedback injection to a small fraction of nodes to carry out the global performances of the total networks. It is a competent and useful strategy especially for the large size networks. The pinning synchronization of neural networks has been generally examined at the present [25-33]. Meanwhile, various selection rules of pinned nodes have been introduced in the existing literatures. The pinned nodes selection rules according to the out-degree and in-degree of the nodes and the synchronization problem were studied for undirected and directed networks which was presented in $[25,26,34]$. The pinning control problem of neural networks was considered and then some unexceptional pinning conditions were found out [27-29], while Chen et al. [35] expressed that, even applying one single pinned node, the whole networks could be controlled as long as the coupling strength was large enough. Furthermore, Wang and Chen [36] summarized that the most highly connected nodes are pinned in order to get the better performance for the undirected networks.

As discussions mentioned above, hybrid adaptive pinning control for FPS of neural networks with mixed timevarying delays and uncertainties asymmetric coupling is an interesting topic for investigating. Therefore, this paper will be focused on this topic in order to facilitate clear comprehension and the purposes of this paper are given as follows:

(i) The mixed time-varying delays with discrete and distributed time-varying delays are considered in the dynamical nodes and in uncertainties asymmetric coupling, simultaneously, which are different from time-delay case in [23, 27-29]. So, our systems are general ones.

(ii) For the control method, FPS is studied by using the nonlinear and adaptive pinning controls and using the nonlinear and adaptive controls which contain error linear term, time-varying delay error linear term, and distributed time-varying delay error linear term.

(iii) The FPS of this paper focuses on how to determine a set of pinned nodes for a linearly coupled delayed neural network with fixed coupling matrices and strength values. Moreover, this paper used random selection of pinning nodes which is different from the pinning control method in $[13,37]$.

Based on constructing a novel Lyapunov-Krasovskii functional, adaptive control technique, the parameter update law, and the technique of dealing with Jensen's and Cauchy inequalities, some novel sufficient conditions for guaranteeing the existence of the FPS of neural networks with mixed time-varying delays and uncertainties asymmetric coupling are derived. Finally, numerical examples are included to show the effectiveness of using the nonlinear and adaptive pinning controls and the nonlinear and adaptive controls.

The rest of the paper is organized as follows. Section 2 provides some mathematical preliminaries and network model. Section 3 presents FPS of neural network with mixed time-varying delays and hybrid uncertainties asymmetric coupling by hybrid adaptive control and hybrid adaptive pinning control, respectively. In Section 4, some numerical examples illustrate given theoretical results. The paper ends with conclusions in Section 5 and cited references.

\section{Model Description and Preliminaries}

Notations. The following notation will be used in this paper: $\mathscr{R}^{n}$ denotes the $n$-dimensional space and $\|\cdot\|$ denotes the Euclidean vector norm; $A^{T}$ denotes the transpose of matrix $A$; $A$ is symmetric if $A=A^{T} ; I_{N}$ denotes an $N$-dimensional identity matrix; for the matrix $A \in \mathscr{R}^{N} \times \mathscr{R}^{N}$, the $i$ th row and the $i$ th column of $A$ are called the $i$ th row-column pair of $A . A_{l} \in \mathscr{R}^{(N-l) \times(N-l)}$ is the minor matrix of $A \in \mathscr{R}^{N \times N}$ by removing arbitrary $l(1 \leq l \leq N)$ row-column pairs of $A$. The symbol $\otimes$ denotes the Kronecker product.

Consider an array of delayed neural networks consisting of $N$ identical nodes with uncertainties asymmetric coupling

$$
\begin{aligned}
\dot{x}_{i}(t)= & -A x_{i}(t)+W_{1} f_{1}\left(x_{i}(t)\right)+W_{2} f_{2}\left(x_{i}(t-h(t))\right) \\
& +W_{3} \int_{t-k(t)}^{t} f_{3}\left(x_{i}(\theta)\right) d \theta \\
& +c_{1} \sum_{j=1}^{N} G_{1_{i j}}\left(\Gamma_{1}+\Delta \Gamma_{1}\right) x_{j}(t) \\
& +c_{2} \sum_{j=1}^{N} G_{2_{i j}}\left(\Gamma_{2}+\Delta \Gamma_{2}\right) x_{j}(t-h(t)) \\
& +c_{3} \sum_{j=1}^{N} G_{3_{i j}}\left(\Gamma_{3}+\Delta \Gamma_{3}\right) \int_{t-k(t)}^{t} x_{j}(\theta) d \theta+\mathcal{U}_{i}(t),
\end{aligned}
$$


where $N$ is the number of coupled nodes, $x_{i}(t)=\left(x_{i 1}(t)\right.$, $\left.x_{i 2}(t), \ldots, x_{i n}(t)\right)^{T} \in \mathscr{R}^{n}$ is the neuron state vector of the $i$ th node, $n$ denotes the number of neurons in a neural network, $A=\operatorname{diag}\left(a_{1}, a_{2}, \ldots, a_{n}\right)>0$ denote the rate with which the cell $i$ resets its potential to the resting state when being isolated from other cells and inputs, $W_{1}, W_{2}$, and $W_{3}$ are connection weight matrices, $h(t)$ and $k(t)$ are the timevarying delays, $f_{r}\left(x_{i}(\cdot)\right)=\left[f_{r 1}\left(x_{i 1}(\cdot)\right), \ldots, f_{r n}\left(x_{i n}(\cdot)\right)\right]^{T}, r=$ $1,2,3$, denote the neuron activation function vector, the positive constants $c_{1}, c_{2}$, and $c_{3}$ are the strength values for the constant coupling and delayed couplings, respectively, $\mathscr{U}_{i}(t) \in \mathscr{R}^{m}$ are the control input of the node $i, \Gamma_{1}, \Gamma_{2}$, and $\Gamma_{3} \in$ $\mathscr{R}^{n \times n}$ are constant inner-coupling matrices and it is assumed that $\Gamma_{1}, \Gamma_{2}$, and $\Gamma_{3}$ are positive definite matrix, $\Delta \Gamma_{1}, \Delta \Gamma_{2}$, and $\Delta \Gamma_{3}$ are the uncertainties of inner-coupling matrices, and $G_{r}=\left(G_{r_{i j}}\right)_{N \times N}(r=1,2,3)$ are the outer-coupling matrices and satisfy the following conditions:

$$
\begin{aligned}
& G_{r_{i j}} \geq 0, \quad i \neq j, r=1,2,3, \\
& G_{r_{i i}}=-\sum_{j=1, j \neq i}^{N} G_{r_{i j}}, \quad i, j=1,2, \ldots, N .
\end{aligned}
$$

Assumption 1. The time-varying delay function $h(t)$ is differential function and $k(t)$ satisfies the conditions $0 \leq h(t) \leq$ $h, 0 \leq k(t) \leq k$, and $0 \leq \dot{h}(t) \leq \mu<1$.

Assumption 2. The activation functions $f_{r i}(\cdot), r=1,2,3, i=$ $1,2, \ldots, n$, satisfy the Lipschitz constants $f_{r i}>0$ :

$$
\begin{aligned}
& \left\|f_{1 i}(x(t))-f_{1 i}(\alpha(t) y(t))\right\| \leq F_{1}\|x(t)-\alpha(t) y(t)\|, \\
& \left\|f_{2 i}(x(t-h(t)))-f_{2 i}(\alpha(t) y(t-h(t)))\right\| \\
& \quad \leq F_{2}\|x(t-h(t))-\alpha(t) y(t-h(t))\|, \\
& \left\|f_{3 i}(x(\theta))-f_{3 i}(\alpha(t) y(\theta))\right\| \\
& \quad \leq F_{3}\|x(\theta)-\alpha(t) y(\theta)\|,
\end{aligned}
$$

where $F_{r}(r=1,2,3)$ are positive constant matrices and we denote

$$
\begin{aligned}
& F_{1}=\operatorname{diag}\left\{f_{1 i}, i=1,2, \ldots, n\right\}, \\
& F_{2}=\operatorname{diag}\left\{f_{2 i}, i=1,2, \ldots, n\right\}, \\
& F_{3}=\operatorname{diag}\left\{f_{3 i}, i=1,2, \ldots, n\right\} .
\end{aligned}
$$

Assumption 3. The parameter uncertainties are assumed to satisfy the following conditions:

$$
\left[\Delta \Gamma_{1}(t) \Delta \Gamma_{2}(t) \Delta \Gamma_{3}(t)\right]=M \nabla(t)\left[\begin{array}{lll}
E_{1} & E_{2} & E_{3}
\end{array}\right],
$$

where $M, E_{1}, E_{2}$, and $E_{3}$ are known real constant matrices and $\nabla(t)$ is and unknown time-varying matrix function satisfying $\nabla^{T}(t) \nabla(t) \leq I$.

The isolated dynamic network is

$$
\begin{aligned}
\dot{s}(t)= & -A s(t)+W_{1} f_{1}(s(t))+W_{2} f_{2}(s(t-h(t))) \\
& +W_{3} \int_{t-k(t)}^{t} f_{3}(s(\theta)) d \theta,
\end{aligned}
$$

where $s(t)=\left(s_{1}(t), s_{2}(t), \ldots, s_{n}(t)\right)^{T} \in \mathscr{R}^{n}$ with $x_{0} \in \mathscr{R}^{n}$ and the parameters $A, W_{1}, W_{2}$, and $W_{3}$ and the nonlinear functions $f(\cdot)$ have the same definitions as in (1).

Definition 4 (FPS). Network (1) with time delay is said to achieve function projective synchronization if there exists a continuously differentiable scaling function $\alpha(t)$ such that

$$
\begin{aligned}
\lim _{t \rightarrow \infty}\left\|e_{i}(t)\right\|=\lim _{t \rightarrow \infty}\left\|x_{i}(t)-\alpha(t) s(t)\right\|, & \\
& i=1,2, \ldots, N,
\end{aligned}
$$

where $\|\cdot\|$ stands for the Euclidean vector norm and $s(t) \in \mathscr{R}^{n}$ can be an equilibrium point, or a (quasi-)periodic orbit, or an orbit of a chaotic attractor.

To investigate the stability of the synchronized states (1), we set the synchronization error $e_{i}(t)$ in the form $e_{i}(t)=$ $x_{i}(t)-\alpha(t) s(t), i=1, \ldots, N$. Then, substituting it into (1), it is easy to get the following:

$$
\begin{aligned}
\dot{e}_{i}(t)= & \dot{x}_{i}(t)-\dot{\alpha}(t) s(t)-\alpha(t) \dot{s}(t) \\
= & -A e_{i}(t)+W_{1}\left[f_{1}\left(x_{i}(t)\right)-\alpha(t) f_{1}(s(t))\right] \\
& +W_{2}\left[f_{2}\left(x_{i}(t-h(t))\right)-\alpha(t) f_{2}(s(t-h(t)))\right] \\
& +W_{3} \int_{t-k(t)}^{t}\left[f_{3}\left(x_{i}(\theta)\right)-\alpha(t) f_{3}(s(\theta))\right] d \theta \\
& +c_{1} \sum_{j=1}^{N} G_{1_{i j}}\left(\Gamma_{1}+\Delta \Gamma_{1}\right) e_{j}(t) \\
& +c_{2} \sum_{j=1}^{N} G_{2_{i j}}\left(\Gamma_{2}+\Delta \Gamma_{2}\right) e_{j}(t-h(t)) \\
& +c_{3} \sum_{j=1}^{N} G_{3_{i j}}\left(\Gamma_{3}+\Delta \Gamma_{3}\right) \int_{t-k(t)}^{t} e_{j}(\theta) d \theta-\dot{\alpha}(t) s(t) \\
& +\mathscr{U}_{i}(t) .
\end{aligned}
$$

The initial condition of (8) is defined by

$$
e_{i}(r)=\phi_{i}(r), \quad-\tau \leq r \leq 0,
$$

where $\tau=\max \{k, h\}$ and $\phi_{i}(r) \in \mathscr{C}\left([-\tau, 0], \mathscr{R}^{n}\right), i=1,2$, $\ldots, N$.

Remark 5. If neural networks (1) are without parameter uncertainties and $c_{3}=0$, the networks model turns into the neural networks proposed in [27-29]:

$$
\begin{aligned}
\dot{x}_{i}(t)= & -A x_{i}(t)+W_{1} f_{1}\left(x_{i}(t)\right) \\
& +W_{2} f_{2}\left(x_{i}(t-h(t))\right) \\
& +W_{3} \int_{t-k(t)}^{t} f_{3}\left(x_{i}(\theta)\right) d \theta+c_{1} \sum_{j=1}^{N} G_{1_{i j}} \Gamma_{1} x_{j}(t) \\
& +c_{2} \sum_{j=1}^{N} G_{2_{i j}} \Gamma_{2} x_{j}(t-h(t)), \quad i=1,2, \ldots, N .
\end{aligned}
$$


Hence, our network model (1) includes previous network model, which can be regarded as a special case of neural network (1).

Lemma 6 (see [5] (Cauchy inequality)). For any symmetric positive definite matrixes $N \in M^{n \times n}$ and $x, y \in \mathscr{R}^{n}$ we have

$$
\pm 2 x^{T} y \leq x^{T} N x+y^{T} N^{-1} y .
$$

Lemma 7 (see [5]). For any constant symmetric matrixes $M \epsilon$ $\mathscr{R}^{m \times m}, M=M^{T}>0$, and $\gamma>0$, vector function $\omega:[0, \gamma] \rightarrow$ $\mathscr{R}^{m}$ such that the integrations concerned are well defined, and then

$$
\begin{gathered}
\left(\int_{0}^{\gamma} \omega^{T}(\theta) d \theta\right)^{T} M\left(\int_{0}^{\gamma} \omega(\theta) d \theta\right) \\
\quad \leq \gamma \int_{0}^{\gamma} \omega^{T}(s) M \omega(\theta) d \theta .
\end{gathered}
$$

Lemma 8 (see [38]). Let $c \in \mathscr{R}$ and $A, B, C$, and $D$ be matrices with appropriate dimensions. Then

(i) $c(A \otimes B)=(c A) \otimes B=A \otimes(c B)$,

(ii) $(A \otimes B)^{T}=A^{T} \otimes B^{T}$,

(iii) $(A \otimes B)(C \otimes D)=(A C) \otimes(B D)$,

(iv) $A \otimes(B+C)=(A \otimes B)+(A \otimes C)$,

(v) $A \otimes B \otimes C=(A \otimes B) \otimes C=A \otimes(B \otimes C)$.

Lemma 9 (see [25]). Assume that $A$ and $B$ are the $N \times N$ Hermitian matrices. Suppose that $\alpha_{1} \geq \alpha_{2} \geq \cdots \geq \alpha_{N}$, $\beta_{1} \geq \beta_{2} \geq \cdots \geq \beta_{N}$, and $\gamma_{1} \geq \gamma_{2} \geq \cdots \geq \gamma_{N}$ are eigenvalues of matrices $A, B$, and $A+B$, respectively. Then one has $\alpha_{i}+\beta_{N} \leq \gamma_{i} \leq \alpha_{i}+\beta_{1}, i=1,2, \ldots, N$.

Lemma 10 (see [35]). If $A=\left(a_{i j}\right)_{N \times N}$ is irreducible and satisfies $a_{i j}=a_{j i} \geq 0, i \neq j, a_{i i}=-\sum_{j=1, i \neq j}^{N} a_{i j}, i, j=$
$1,2, \ldots, N$. Then, for any constant $\xi>0$, all eigenvalues of the matrix $A-\Xi$ are negative definite, where $\Xi=\operatorname{diag}(\xi, 0, \ldots, 0)$.

Lemma 11 (see [25]). For a symmetric matrix $M \in \mathscr{R}^{N \times N}$ and a diagonal matrix $D=\operatorname{diag}(d_{1}, \ldots, d_{l}, \underbrace{0, \ldots, 0}_{N-l})$ with $d_{i}>$ $0, i=1,2, \ldots, l(1 \leq l<N)$, let

$$
M-D=\left[\begin{array}{cc}
A-\widetilde{D} & B \\
B^{T} & M_{l}
\end{array}\right],
$$

where $M_{l}$ is the minor matrix of $M$ by removing its first $l$ row-column pairs, $A$ and $B$ are matrices with appropriate dimensions, and $\widetilde{D}=\operatorname{diag}\left(d_{1}, \ldots, d_{l}\right)$. If $d_{i}>\lambda_{\max }(A-$ $\left.B M_{l}^{-1} B^{T}\right), i=1, \ldots, l, M-D<0$ is equivalent to $M_{l}<0$.

\section{Main Results}

In this section, we present hybrid control scheme to synchronize neural networks (1) to the homogenous trajectory (6). Then, we will give some sufficient conditions in FPS of neural networks with mixed time-varying delays and uncertainties asymmetric coupling.

3.1. FPS under Hybrid Adaptive Pinning Control. We design nonlinear and adaptive pinning controls to realize FPS of neural networks with mixed time-varying delays and uncertainties asymmetric coupling. In order to stabilize the origin of neural networks (1) by means of nonlinear and adaptive pinning controls $\mathscr{U}_{i}(t)$ such as

$$
\mathscr{U}_{i}(t)=u_{i 1}(t)+u_{i 2}(t), \quad i=1,2, \ldots, N
$$

where

$$
\begin{aligned}
& u_{i 1}(t)=\dot{\alpha}(t) s(t)-W_{1}\left[\alpha(t) f_{1}(s(t))+f_{1}(\alpha(t) s(t))\right]-W_{2}\left[\alpha(t) f_{2}(s(t-h(t)))+f_{2}(\alpha(t) s(t-h(t)))\right] \\
& -W_{3} \int_{t-k(t)}^{t}\left[\alpha(t) f_{3}(s(\theta))-f_{3}(\alpha(t) s(\theta))\right] d \theta, \quad i=1,2, \ldots, N, \\
& u_{i 2}(t)= \begin{cases}-c_{1} d_{1 i}(t) \Gamma_{1} e_{i}(t)-c_{2} d_{2 i}(t) \Gamma_{2} e_{i}(t-h(t))-c_{3} d_{3 i}(t) \Gamma_{3} \int_{t-k(t)}^{t} e_{i}(\theta) d \theta, & i=1,2, \ldots, l, \\
0 & i=l+1, l+2, \ldots, N,\end{cases}
\end{aligned}
$$

the updating laws are

$$
\begin{aligned}
& \dot{d}_{1 i}(t)=\beta_{i 1} e_{i}^{T}(t) \Gamma_{1} e_{i}(t), \\
& \dot{d}_{2 i}(t)=\beta_{i 2} e_{i}^{T}(t) \Gamma_{2} e_{i}(t-h(t)), \\
& \dot{d}_{3 i}(t)=\beta_{i 3} e_{i}^{T}(t) \Gamma_{3} \int_{t-k(t)}^{t} e_{i}(\theta) d \theta,
\end{aligned}
$$

where $\beta_{i 1}, \beta_{i 2}$, and $\beta_{i 3}$ are positive constants and $s(t)$ is a solution of an isolated node. The controller $\mathcal{U}_{i}(t)$ is different type of controller, and $u_{i 1}(t)$ is the nonlinear control and $u_{i 2}(t)$ is the adaptive linear pinning control. Then, substituting (14) into (8), it can be derived that

$$
\begin{aligned}
\dot{e}_{i}(t)= & -A e_{i}(t)+W_{1} \tilde{f}_{1}\left(e_{i}(t)\right) \\
& +W_{2} \tilde{f}_{2}\left(e_{i}(t-h(t))\right)
\end{aligned}
$$




$$
\begin{aligned}
& +W_{3} \int_{t-k(t)}^{t} \widetilde{f}_{3}\left(e_{i}(\theta)\right) d \theta \\
& +c_{1} \sum_{j=1}^{N} G_{1_{i j}}\left(\Gamma_{1}+\Delta \Gamma_{1}\right) e_{j}(t) \\
& +c_{2} \sum_{j=1}^{N} G_{2_{i j}}\left(\Gamma_{2}+\Delta \Gamma_{2}\right) e_{j}(t-h(t)) \\
& +c_{3} \sum_{j=1}^{N} G_{3_{i j}}\left(\Gamma_{3}+\Delta \Gamma_{3}\right) \int_{t-k(t)}^{t} e_{j}(\theta) d \theta \\
& -c_{1} d_{1 i}(t) \Gamma_{1} e_{i}(t) \\
& -c_{2} d_{2 i}(t) \Gamma_{2} e_{i}(t-h(t)) \\
& -c_{3} d_{3 i}(t) \Gamma_{3} \int_{t-k(t)}^{t} e_{i}(\theta) d \theta \text {, } \\
& i=1,2, \ldots, l, \\
& \dot{e}_{i}(t)=-A e_{i}(t)+W_{1} \tilde{f}_{1}\left(e_{i}(t)\right) \\
& +W_{2} \widetilde{f}_{2}\left(e_{i}(t-h(t))\right) \\
& +W_{3} \int_{t-k(t)}^{t} \tilde{f}_{3}\left(e_{i}(\theta)\right) d \theta \\
& +c_{1} \sum_{j=1}^{N} G_{1_{i j}}\left(\Gamma_{1}+\Delta \Gamma_{1}\right) e_{j}(t) \\
& +c_{2} \sum_{j=1}^{N} G_{2_{i j}}\left(\Gamma_{2}+\Delta \Gamma_{2}\right) e_{j}(t-h(t)) \\
& +c_{3} \sum_{j=1}^{N} G_{3_{i j}}\left(\Gamma_{3}+\Delta \Gamma_{3}\right) \int_{t-k(t)}^{t} e_{j}(\theta) d \theta, \\
& i=l+1, l+2, \ldots, N, \\
& \dot{d}_{1 i}(t)=\beta_{i 1} e_{i}^{T}(t) \Gamma_{1} e_{i}(t), \quad i=1,2, \ldots, l, \\
& \dot{d}_{2 i}(t)=\beta_{i 2} e_{i}^{T}(t) \Gamma_{2} e_{i}(t-h(t)), \quad i=1,2, \ldots, l, \\
& \dot{d}_{3 i}(t)=\beta_{i 3} e_{i}^{T}(t) \Gamma_{3} \int_{t-k(t)}^{t} e_{i}(\theta) d \theta, \quad i=1,2, \ldots, l,
\end{aligned}
$$

where

$$
\begin{aligned}
\tilde{f}_{1}\left(e_{i}(t)\right)= & f_{1}\left(x_{i}(t)\right)-f_{1}(\alpha(t) s(t)), \\
\tilde{f}_{2}\left(e_{i}(t-h(t))\right)= & f_{2}\left(x_{i}(t-h(t))\right) \\
& -f_{2}(\alpha(t) s(t-h(t))), \\
\tilde{f}_{3}\left(e_{i}(\theta)\right)= & f_{3}\left(x_{i}(\theta)\right)-f_{3}(\alpha(t) s(\theta)),
\end{aligned}
$$

and, for convenience, we denote

$$
\begin{aligned}
& \Pi_{1}=\frac{1}{\lambda_{\min }\left(I_{N} \otimes \Gamma_{1}\right)}\left[-\lambda_{\max }\left(I_{N} \otimes A\right)\right. \\
& +\frac{1}{2 \varepsilon_{1}} \lambda_{\max }\left(I_{N} \otimes W_{1} W_{1}^{T}\right)+\frac{\varepsilon_{1}}{2} \lambda_{\max }\left(I_{N} \otimes F_{1}^{T} F_{1}\right) \\
& +\frac{c_{3} k^{2}}{2} \lambda_{\max }\left(I_{N} \otimes \Gamma_{3}\right)+\frac{1}{2 \varepsilon_{2}} \lambda_{\max }\left(I_{N} \otimes W_{2} W_{2}^{T}\right) \\
& +\frac{1}{2 \varepsilon_{3}} \lambda_{\max }\left(I_{N} \otimes W_{3} W_{3}^{T}\right)+\frac{c_{1} \varepsilon_{6}}{2} \lambda_{\max }\left(I_{N} \otimes E_{1}^{T} E_{1}\right) \\
& +\frac{c_{1}}{2 \varepsilon_{6}} \lambda_{\max }\left(G_{1} G_{1}^{T}\right) \lambda_{\max }\left(M M^{T}\right) \\
& +\frac{c_{2}}{2 \varepsilon_{7}} \lambda_{\max }\left(G_{2} G_{2}^{T}\right) \lambda_{\max }\left(\Gamma_{2} \Gamma_{2}^{T}\right) \\
& +\frac{c_{2}}{2 \varepsilon_{8}} \lambda_{\max }\left(G_{2} G_{2}^{T}\right) \lambda_{\max }\left(M M^{T}\right) \\
& +\frac{c_{3}}{2 \varepsilon_{9}} \lambda_{\max }\left(G_{3} G_{3}^{T}\right) \lambda_{\max }\left(\Gamma_{3} \Gamma_{3}^{T}\right) \\
& +\frac{c_{3}}{2 \varepsilon_{10}} \lambda_{\max }\left(G_{3} G_{3}^{T}\right) \lambda_{\max }\left(M M^{T}\right) \\
& +\frac{c_{2}}{2(1-\mu)} \lambda_{\max }\left(I_{N} \otimes \Gamma_{2}\right) \\
& +\frac{c_{2} \bar{d}_{2}^{*}}{2 \varepsilon_{4}} \lambda_{\max }\left(I_{N} \otimes \Gamma_{2} \Gamma_{2}^{T}\right) \\
& \left.+\frac{c_{3} \bar{d}_{3}^{*}}{2 \varepsilon_{5}} \lambda_{\max }\left(I_{N} \otimes \Gamma_{3} \Gamma_{3}^{T}\right)\right], \\
& \Pi_{2}=\frac{1}{2 \lambda_{\min }\left(I_{N} \otimes \Gamma_{2}\right)}\left[\varepsilon_{2} \lambda_{\max }\left(I_{N} \otimes F_{2}^{T} F_{2}\right)+c_{2} \varepsilon_{7}\right. \\
& \left.+c_{2} \varepsilon_{8} \lambda_{\max }\left(I_{N} \otimes E_{2}^{T} E_{2}\right)+c_{2} \bar{d}_{2}^{*} \varepsilon_{4}\right], \\
& \Pi_{3}=\frac{1}{2 \lambda_{\min }\left(I_{N} \otimes \Gamma_{3}\right)}\left[\varepsilon_{3} \lambda_{\text {max }}\left(I_{N} \otimes F_{3}^{T} F_{3}\right)+c_{3} \varepsilon_{9}\right. \\
& \left.+c_{3} \varepsilon_{10} \lambda_{\max }\left(I_{N} \otimes E_{3}^{T} E_{3}\right)+c_{3} \bar{d}_{3}^{*} \varepsilon_{5}\right] \text {, } \\
& \xi(t)=\left(e^{T}(t), e^{T}(t-h(t)),\left(\int_{t-k(t)}^{t} e(\theta) d \theta\right)^{T}\right)^{T} .
\end{aligned}
$$

Theorem 12. For some given synchronization scaling function $\alpha(t)$, neural networks (1) satisfying Assumptions 1, 2, and 3, and target system can realize function projective synchronization by the nonlinear and adaptive pinning control law as shown in (14) if there exist positive constants $\varepsilon_{i}, i=1,2, \ldots, 10$, and by taking appropriate $d_{1 i}^{*}(i=1,2, \ldots, l), \bar{d}_{2}^{*}$, and $\bar{d}_{3}^{*}$ such that

$$
\lambda_{\max }\left(\frac{G_{1}+G_{1}^{T}}{2}\right)_{l}<-\frac{\Pi_{1}}{c_{1}},
$$




$$
\begin{aligned}
\bar{d}_{2}^{*} & -\frac{1}{c_{2} \varepsilon_{4}}\left[\varepsilon_{2} \lambda_{\max }\left(I_{N} \otimes F_{2}^{T} F_{2}\right)+c_{2} \varepsilon_{7}\right. \\
& \left.+c_{2} \varepsilon_{8} \lambda_{\max }\left(I_{N} \otimes E_{2}^{T} E_{2}\right)-\lambda_{\min }\left(I_{N} \otimes \Gamma_{2}\right)\right]>0, \\
\bar{d}_{3}^{*} & -\frac{1}{c_{3} \varepsilon_{5}}\left[\varepsilon_{3} \lambda_{\max }\left(I_{N} \otimes F_{3}^{T} F_{3}\right)+c_{3} \varepsilon_{9}\right. \\
& \left.+c_{3} \varepsilon_{10} \lambda_{\max }\left(I_{N} \otimes E_{3}^{T} E_{3}\right)-\lambda_{\min }\left(I_{N} \otimes \Gamma_{3}\right)\right]>0 .
\end{aligned}
$$

Then controlled neural network (1) is function projective synchronization.

Proof. Construct the following Lyapunov-Krasovskii functional candidate:

$$
V(t)=V_{1}(t)+V_{2}(t)+V_{3}(t)+V_{4}(t)
$$

where

$$
\begin{aligned}
V_{1}(t)= & \frac{1}{2} \sum_{i=1}^{N} e_{i}^{T}(t) e_{i}(t), \\
V_{2}(t)= & \frac{c_{2}}{2(1-\mu)} \sum_{i=1}^{N} \int_{t-h(t)}^{t} e_{i}^{T}(\theta) \Gamma_{2} e_{i}(\theta) d \theta, \\
V_{3}(t)= & \frac{c_{3} k}{2} \sum_{i=1}^{N} \int_{-k}^{0} \int_{t+s}^{t} e_{i}^{T}(\theta) \Gamma_{3} e_{i}(\theta) d \theta d s, \\
V_{4}(t)= & \frac{c_{1}}{2} \sum_{i=1}^{l} \frac{1}{\beta_{i 1}}\left(d_{1 i}(t)-d_{1 i}^{*}\right)^{2} \\
& +\frac{c_{2}}{2} \sum_{i=1}^{l} \frac{1}{\beta_{i 2}}\left(d_{2 i}(t)-d_{2 i}^{*}\right)^{2} \\
& +\frac{c_{3}}{2} \sum_{i=1}^{l} \frac{1}{\beta_{i 3}}\left(d_{3 i}(t)-d_{3 i}^{*}\right)^{2} .
\end{aligned}
$$

By taking the derivative of $V(t)$ along the trajectories of system (17), we have the following:

$$
\begin{aligned}
& \dot{V}_{1}(t)=-\sum_{i=1}^{N} e_{i}^{T}(t) A e_{i}(t)+\sum_{i=1}^{N} e_{i}^{T}(t) W_{1} \tilde{f}_{1}\left(e_{i}(t)\right) \\
& +\sum_{i=1}^{N} e_{i}^{T}(t) W_{2} \tilde{f}_{2}\left(e_{i}(t-h(t))\right) \\
& +\sum_{i=1}^{N} e_{i}^{T}(t) W_{3} \int_{t-k(t)}^{t} \tilde{f}_{3}\left(e_{i}(\theta)\right) d \theta \\
& +c_{1} \sum_{i=1}^{N} \sum_{j=1}^{N} e_{i}^{T}(t) G_{1_{i j}}\left(\Gamma_{1}+\Delta \Gamma_{1}\right) e_{j}(t) \\
& +c_{2} \sum_{i=1}^{N} \sum_{j=1}^{N} e_{i}^{T}(t) G_{2_{i j}}\left(\Gamma_{2}+\Delta \Gamma_{2}\right) e_{j}(t-\tau(t)) \\
& +c_{3} \sum_{i=1}^{N} \sum_{j=1}^{N} e_{i}^{T}(t) G_{3_{i j}}\left(\Gamma_{3}+\Delta \Gamma_{3}\right) \int_{t-k(t)}^{t} e_{j}(\theta) d \theta
\end{aligned}
$$

$$
\begin{aligned}
& -\sum_{i=1}^{N} e_{i}^{T}(t) c_{1} d_{1 i}(t) \Gamma_{1} e_{i}(t) \\
& -\sum_{i=1}^{N} e_{i}^{T}(t) c_{2} d_{2 i}(t) \Gamma_{2} e_{i}(t-h(t)) \\
& -\sum_{i=1}^{N} e_{i}^{T}(t) c_{3} d_{3 i}(t) \Gamma_{3} \int_{t-k(t)}^{t} e_{i}(\theta) d \theta .
\end{aligned}
$$

$$
\begin{aligned}
\dot{V}_{2}(t) & =\frac{c_{2}}{2(1-\mu)} \sum_{i=1}^{N} e_{i}^{T}(t) \Gamma_{2} e_{i}(t) \\
& -\frac{c_{2}(1-\dot{h}(t))}{2(1-\mu)} \sum_{i=1}^{N} e_{i}^{T}(t-h(t)) \Gamma_{2} e_{i}(t-h(t)) .
\end{aligned}
$$

$$
\begin{aligned}
\dot{V}_{3}(t) & =\frac{c_{3} k^{2}}{2} \sum_{i=1}^{N} e_{i}^{T}(t) \Gamma_{3} e_{i}(t) \\
& -\frac{c_{3} k}{2} \sum_{i=1}^{N} \int_{t-k(t)}^{t} e_{i}^{T}(\theta) \Gamma_{3} e_{i}(\theta) d \theta .
\end{aligned}
$$$$
\dot{V}_{4}(t)=\sum_{i=1}^{l} \frac{c_{1}}{\beta_{i 1}}\left(d_{1 i}(t)-d_{1 i}^{*}\right) \beta_{i 1} e_{i}^{T}(t) \Gamma_{1} e_{i}(t)
$$$$
+\sum_{i=1}^{l} \frac{c_{2}}{\beta_{i 2}}\left(d_{2 i}(t)-d_{2 i}^{*}\right) \beta_{i 2} e_{i}^{T}(t) \Gamma_{2} e_{i}(t-h(t))
$$$$
+\sum_{i=1}^{l} \frac{c_{3}}{\beta_{i 3}}\left(d_{3 i}(t)-d_{3 i}^{*}\right) \beta_{i 3} e_{i}^{T}(t) \Gamma_{3} \int_{t-k(t)}^{t} e_{i}(\theta) d \theta .
$$

After $V(t)$ were calculated, we will get that

$$
\begin{aligned}
& \dot{V}(t) \leq-\sum_{i=1}^{N} e_{i}^{T}(t) A e_{i}(t)+\sum_{i=1}^{N} e_{i}^{T}(t) W_{1} \tilde{f}_{1}\left(e_{i}(t)\right) \\
& +\sum_{i=1}^{N} e_{i}^{T}(t) W_{2} \tilde{f}_{2}\left(e_{i}(t-h(t))\right)+\sum_{i=1}^{N} e_{i}^{T}(t) W_{3} \\
& \cdot \int_{t-k(t)}^{t} \tilde{f}_{3}\left(e_{i}(\theta)\right) d \theta \\
& +c_{1} \sum_{i=1}^{N} \sum_{j=1}^{N} e_{i}^{T}(t) G_{1_{i j}}\left(\Gamma_{1}+\Delta \Gamma_{1}\right) e_{j}(t) \\
& +c_{2} \sum_{i=1}^{N} \sum_{j=1}^{N} e_{i}^{T}(t) G_{2_{i j}}\left(\Gamma_{2}+\Delta \Gamma_{2}\right) e_{j}(t-\tau(t)) \\
& +c_{3} \sum_{i=1}^{N} \sum_{j=1}^{N} e_{i}^{T}(t) G_{3_{i j}}\left(\Gamma_{3}+\Delta \Gamma_{3}\right) \times \int_{t-k(t)}^{t} e_{j}(\theta) d \theta \\
& +\frac{c_{2}}{2(1-\mu)} \sum_{i=1}^{N} e_{i}^{T}(t) \Gamma_{2} e_{i}(t)-\frac{c_{2}}{2(1-\mu)}
\end{aligned}
$$




$$
\begin{aligned}
& \cdot \sum_{i=1}^{N} e_{i}^{T}(t-h(t)) \Gamma_{2} e_{i}(t-h(t))+\frac{c_{3} k^{2}}{2} \\
& \cdot \sum_{i=1}^{N} e_{i}^{T}(t) \Gamma_{3} e_{i}(t)-\frac{c_{3} k}{2} \sum_{i=1}^{N} \int_{t-k(t)}^{t} e_{i}^{T}(\theta) \Gamma_{3} e_{i}(\theta) d \theta \\
& -c_{1} \sum_{i=1}^{l} d_{1 i}^{*} e_{i}^{T}(t) \Gamma_{1} e_{i}(t) \\
& -c_{2} \sum_{i=1}^{l} d_{2 i}^{*} e_{i}^{T}(t) \Gamma_{2} e_{i}(t-h(t)) d s \\
& -c_{3} \sum_{i=1}^{l} d_{3 i}^{*} i_{i}^{T}(t) \Gamma_{3} \int_{t-k(t)}^{t} e_{i}(\theta) d \theta \\
& \leq-e^{T}(t)\left(I_{N} \otimes A\right) e(t)+e^{T}(t)\left(I_{N} \otimes W_{1}\right) \tilde{f}_{1}(e(t)) \\
& +e^{T}(t)\left(I_{N} \otimes W_{2}\right) \tilde{f}_{2}(e(t-h(t)))+e^{T}(t)\left(I_{N}\right. \\
& \left.\otimes W_{3}\right) \int_{t-k(t)}^{t} \tilde{f}_{3}(e(\theta)) d \theta+c_{1} e^{T}(t)\left(G_{1}\right. \\
& \left.\otimes\left(\Gamma_{1}+\Delta \Gamma_{1}\right)\right) e(t)+c_{2} e^{T}(t)\left(G_{2} \otimes\left(\Gamma_{2}+\Delta \Gamma_{2}\right)\right) e(t \\
& -h(t))+c_{3} e^{T}(t)\left(G_{3} \otimes\left(\Gamma_{3}+\Delta \Gamma_{3}\right)\right) \int_{t-k(t)}^{t} e(\theta) d \theta \\
& +\frac{\mathcal{C}_{2}}{2(1-\mu)} e^{T}(t)\left(I_{N} \otimes \Gamma_{2}\right) e(t)-\frac{\mathcal{C}_{2}}{2(1-\mu)} e^{T}(t \\
& -h(t))\left(I_{N} \otimes \Gamma_{2}\right) e(t-h(t))+\frac{c_{3} k^{2}}{2} e^{T}(t)\left(I_{N} \otimes \Gamma_{3}\right) \\
& \cdot e(t)-\frac{c_{3} k}{2} \int_{t-k(t)}^{t} e^{T}(\theta)\left(I_{N} \otimes \Gamma_{3}\right) e(\theta) d \theta \\
& -c_{1} e^{T}(t)\left(D_{1}^{*} \otimes \Gamma_{1}\right) e(t)-c_{2} \bar{d}_{2}^{*} e^{T}(t)\left(I_{N} \otimes \Gamma_{2}\right) \\
& \cdot e(t-h(t))-c_{3} \bar{d}_{3}^{*} e^{T}(t)\left(I_{N} \otimes \Gamma_{3}\right) \int_{t-k(t)}^{t} e(\theta) d \theta
\end{aligned}
$$

where $e(t)=\left(e_{1}(t), \ldots, e_{N}(t)\right) \in \mathscr{R}^{n \times N}, e(t-h(t))=$ $\left(e_{1}(t-h(t)), \ldots, e_{N}(t-h(t))\right) \in \mathscr{R}^{n \times N}, \int_{t-k(t)}^{t} e(\theta) d \theta=$ $\int_{t-k(t)}^{t}\left(e_{1}(\theta), e_{2}(\theta), \ldots, e_{N}(\theta)\right) d \theta \in \mathscr{R}^{n \times N}, D_{1}^{*}=\operatorname{diag}\left(d_{11}^{*}\right.$, $\left.d_{12}^{*}, \ldots, d_{1 l}^{*}, 0, \ldots, 0\right) \in \mathscr{R}^{N \times N}, \bar{d}_{2}^{*}=\max _{1 \leq i \leq l}\left\{d_{2 i}^{*}\right\}$, and $\bar{d}_{3}^{*}=$ $\max _{1 \leq i \leq l}\left\{d_{3 i}^{*}\right\}$.

From Assumption 2, we obtain the following three inequalities:

$$
\begin{aligned}
& \left\|\tilde{f}_{1 i}\left(e_{i}(t)\right)\right\|=\left\|\tilde{f}_{1 i}(x(t))-\tilde{f}_{1 i}(\alpha(t) s(t))\right\| \\
& \quad \leq F_{1 i}\left\|e_{i}(t)\right\|, \\
& \left\|\tilde{f}_{2 i}\left(e_{i}(t-h(t))\right)\right\| \\
& \quad=\left\|\tilde{f}_{2 i}(x(t-h(t)))-\tilde{f}_{2 i}(\alpha(t) s(t-h(t)))\right\|
\end{aligned}
$$

$$
\begin{aligned}
& \quad \leq F_{2 i}\left\|e_{i}(t-h(t))\right\|, \\
& \left\|\tilde{f}_{3 i}\left(e_{i}(\theta)\right)\right\|=\left\|\tilde{f}_{3 i}(x(\theta))-\tilde{f}_{3 i}(\alpha(t) s(\theta))\right\| \\
& \quad \leq F_{3 i}\left\|e_{i}(\theta)\right\| .
\end{aligned}
$$

Applying Lemmas 6 and 7, we have

$$
\begin{aligned}
& e^{T}(t)\left(I_{N} \otimes W_{1}\right) \tilde{f}_{1}(e(t)) \leq \frac{1}{2 \varepsilon_{1}} e^{T}(t)\left(I_{N} \otimes W_{1} W_{1}^{T}\right) \\
& \cdot e(t)+\frac{\varepsilon_{1}}{2} \tilde{f}_{1}^{T}(e(t))\left(I_{N} \otimes I_{n}\right) \tilde{f}_{1}(e(t)), \\
& \leq \frac{1}{2 \varepsilon_{1}} e^{T}(t)\left(I_{N} \otimes W_{1} W_{1}^{T}\right) e(t)+\frac{\varepsilon_{1}}{2} e^{T}(t) \\
& \cdot\left(I_{N} \otimes F_{1}^{T} F_{1}\right) e(t), \\
& e^{T}(t)\left(I_{N} \otimes W_{2}\right) \tilde{f}_{2}(e(t-h(t))) \leq \frac{1}{2 \varepsilon_{2}} e^{T}(t) \\
& \cdot\left(I_{N} \otimes W_{2} W_{2}^{T}\right) e(t)+\frac{\varepsilon_{2}}{2} \tilde{f}_{2}^{T}((t-h(t)))\left(I_{N} \otimes I_{n}\right) \\
& \cdot \tilde{f}_{2}(e(t-h(t))), \\
& \leq \frac{1}{2 \varepsilon_{2}} e^{T}(t)\left(I_{N} \otimes W_{2} W_{2}^{T}\right) e(t)+\frac{\varepsilon_{2}}{2} e^{T}(t-h(t)) \\
& \cdot\left(I_{N} \otimes F_{2}^{T} F_{2}\right) e(t-h(t)), \\
& e^{T}(t)\left(I_{N} \otimes W_{3}\right) \int_{t-k(t)}^{t} \tilde{f}_{3}(e(\theta)) d \theta \leq \frac{1}{2 \varepsilon_{3}} e^{T}(t) \\
& \quad \cdot\left(I_{N} \otimes W_{3} W_{3}^{T}\right) e(t)+\frac{\varepsilon_{3}}{2}\left(\int_{t-k(t)}^{t} \widetilde{f}_{3}^{T}(e(\theta)) d \theta\right)^{T} \\
& \cdot\left(I_{N} \otimes I_{n}\right)\left(\int_{t-k(t)}^{t} \tilde{f}_{3}^{T}(e(\theta)) d \theta\right), \\
& \frac{1}{2 \varepsilon_{3}} e^{T}(t)\left(I_{N} \otimes W_{3} W_{3}^{T}\right) e(t)+\frac{\varepsilon_{3}}{2}
\end{aligned}
$$

$$
\begin{aligned}
& \times\left(\int_{t-k(t)}^{t} e^{T}(\theta) d \theta\right)^{T}\left(I_{N} \otimes F_{3}^{T} F_{3}\right) \\
& \cdot\left(\int_{t-k(t)}^{t} e^{T}(\theta) d \theta\right)
\end{aligned}
$$$$
-c_{2} \bar{d}_{2}^{*} e^{T}(t)\left(I_{N} \otimes \Gamma_{2}\right) e(t-h(t)) \leq \frac{c_{2} \bar{d}_{2}^{*}}{2 \varepsilon_{4}} e^{T}(t)
$$$$
\cdot\left(I_{N} \otimes \Gamma_{2} \Gamma_{2}^{T}\right) e(t)+\frac{c_{2} \bar{d}_{2}^{*} \varepsilon_{4}}{2} e^{T}(t-h(t))\left(I_{N} \otimes I_{n}\right)
$$$$
\cdot e(t-h(t))
$$

$-c_{3} \bar{d}_{3}^{*} e^{T}(t)\left(I_{N} \otimes \Gamma_{3}\right) \int_{t-k(t)}^{t} e(\theta) d \theta \leq \frac{c_{3} \bar{d}_{3}^{*}}{2 \varepsilon_{5}} e^{T}(t)$ 


$$
\begin{aligned}
& \cdot\left(I_{N} \otimes \Gamma_{3} \Gamma_{3}^{T}\right) e(t)+\frac{c_{3} \bar{d}_{3}^{*} \varepsilon_{5}}{2} \times\left(\int_{t-k(t)}^{t} e^{T}(\theta) d \theta\right)^{T} \\
& \cdot\left(I_{N} \otimes I_{n}\right)\left(\int_{t-k(t)}^{t} e^{T}(\theta) d \theta\right) \\
& c_{1} e^{T}(t)\left(G_{1} \otimes\left(\Gamma_{1}+\Delta \Gamma_{1}\right)\right) e(t)=c_{1} e^{T}(t)\left(G_{1} \otimes \Gamma_{1}\right) \\
& \cdot e(t)+c_{1} e^{T}(t)\left(G_{1} \otimes M \nabla(t) E_{1}\right) e(t), \\
& \leq c_{1} e^{T}(t)\left(G_{1} \otimes \Gamma_{1}\right) e(t)+\frac{c_{1}}{2 \varepsilon_{6}} e^{T}(t)\left(G_{1} G_{1}^{T} \otimes M M^{T}\right) \\
& \cdot e(t)+\frac{c_{1} \varepsilon_{6}}{2} e^{T}(t)\left(I_{N} \otimes E_{1}^{T} E_{1}\right) e(t) \\
& c_{2} e^{T}(t)\left(G_{2} \otimes\left(\Gamma_{2}+\Delta \Gamma_{2}\right)\right) e(t-h(t))=c_{2} e^{T}(t) \\
& \cdot\left(G_{2} \otimes \Gamma_{2}\right) e(t-h(t))+c_{2} e^{T}(t)\left(G_{2} \otimes M \nabla(t) E_{2}\right) \\
& \cdot e(t-h(t)) \text {, } \\
& \leq \frac{c_{2}}{2 \varepsilon_{7}} e^{T}(t)\left(G_{2} G_{2}^{T} \otimes \Gamma_{2} \Gamma_{2}^{T}\right) e(t)+\frac{c_{2} \varepsilon_{7}}{2} e^{T}(t-h(t)) \\
& \cdot\left(I_{N} \otimes I_{n}\right) e(t-h(t))+\frac{c_{2}}{2 \varepsilon_{8}} e^{T}(t) \\
& \cdot\left(G_{2} G_{2}^{T} \otimes M M^{T}\right) e(t)+\frac{c_{2} \varepsilon_{8}}{2} e^{T}(t-h(t)) \\
& \cdot\left(I_{N} \otimes E_{2}^{T} E_{2}\right)(t-h(t)) \text {, } \\
& c_{3} e^{T}(t)\left(G_{3} \otimes\left(\Gamma_{3}+\Delta \Gamma_{3}\right)\right) \int_{t-k(t)}^{t} e(\theta) d \theta=c_{3} e^{T}(t) \\
& \cdot\left(G_{3} \otimes \Gamma_{3}\right) \int_{t-k(t)}^{t} e(\theta) d \theta+c_{3} e^{T}(t) \\
& \cdot\left(G_{3} \otimes M \nabla(t) E_{3}\right) \int_{t-k(t)}^{t} e(\theta) d \theta \\
& \leq \frac{c_{3}}{2 \varepsilon_{9}} e^{T}(t)\left(G_{3} G_{3}^{T} \otimes \Gamma_{3} \Gamma_{3}^{T}\right) e(t) \\
& +\frac{c_{3} \varepsilon_{9}}{2}\left(\int_{t-k(t)}^{t} e^{T}(\theta) d \theta\right)^{T}\left(I_{N} \otimes I_{n}\right) \\
& \cdot\left(\int_{t-k(t)}^{t} e^{T}(\theta) d \theta\right)+\frac{c_{3}}{2 \varepsilon_{10}} e^{T}(t)\left(G_{3} G_{3}^{T} \otimes M M^{T}\right) \\
& \cdot e(t)+\frac{c_{3} \varepsilon_{10}}{2} \times\left(\int_{t-k(t)}^{t} e^{T}(\theta) d \theta\right)^{T}\left(I_{N} \otimes E_{3}^{T} E_{3}\right) \\
& \cdot\left(\int_{t-k(t)}^{t} e^{T}(\theta) d \theta\right)
\end{aligned}
$$

Therefore, we have

$$
e^{T}(t)\left[-c_{1}\left(D_{1}^{*} \otimes \Gamma_{1}\right)+c_{1}\left(G_{1} \otimes \Gamma_{1}\right)+\frac{c_{3} k^{2}}{2}\left(I_{N} \otimes \Gamma_{3}\right)\right.
$$

$$
\begin{aligned}
& +\frac{1}{2 \varepsilon_{2}}\left(I_{N} \otimes W_{2} W_{2}^{T}\right)+\frac{1}{2 \varepsilon_{3}}\left(I_{N} \otimes W_{3} W_{3}^{T}\right) \\
& +\frac{c_{1}}{2 \varepsilon_{6}}\left(G_{1} G_{1}^{T} \otimes M M^{T}\right)+\frac{c_{1} \varepsilon_{6}}{2}\left(I_{N} \otimes E_{1}^{T} E_{1}\right) \\
& +\frac{c_{2}}{2 \varepsilon_{7}}\left(G_{2} G_{2}^{T} \otimes \Gamma_{2} \Gamma_{2}^{T}\right)+\frac{c_{2}}{2 \varepsilon_{8}}\left(G_{2} G_{2}^{T} \otimes M M^{T}\right) \\
& +\frac{c_{3}}{2 \varepsilon_{9}}\left(G_{3} G_{3}^{T} \otimes \Gamma_{3} \Gamma_{3}^{T}\right)+\frac{c_{3}}{2 \varepsilon_{10}}\left(G_{3} G_{3}^{T} \otimes M M^{T}\right) \\
& \left.+\frac{c_{2} \bar{d}_{2}^{*}}{2 \varepsilon_{4}}\left(I_{N} \otimes \Gamma_{2} \Gamma_{2}^{T}\right)+\frac{c_{3} \bar{d}_{3}^{*}}{2 \varepsilon_{5}}\left(I_{N} \otimes \Gamma_{3} \Gamma_{3}^{T}\right)\right] e(t) \\
& -e^{T}(t-h(t))\left[\frac{c_{2}}{2}\left(I_{N} \otimes \Gamma_{2}\right)-\Pi_{2}\left(I_{N} \otimes \Gamma_{2}\right)\right] e(t \\
& -h(t))-\int_{t-k(t)}^{t} e^{T}(\theta) \\
& \cdot\left[\frac{c_{3} k}{2}\left(I_{N} \otimes \Gamma_{3}\right)-\Pi_{3}\left(I_{N} \otimes \Gamma_{3}\right)\right] e(\theta) d \theta, \\
& \leq e^{T}(t)\left[\left(\Pi_{1} I_{N}+c_{1} G_{1}-c_{1} D_{1}^{*}\right) \otimes \Gamma_{1}\right] e(t)-e^{T}(t \\
& -h(t))\left[\left(\frac{c_{2}}{2}-\Pi_{2}\right)\left(I_{N} \otimes \Gamma_{2}\right)\right] e(t-h(t)) \\
& -\int_{t-k(t)}^{t} e^{T}(\theta)\left[\left(\frac{c_{3} k}{2}-\Pi_{3}\right)\left(I_{N} \otimes \Gamma_{3}\right)\right] e(\theta) d \theta, \\
& =e^{T}(t)\left[\left(\mathscr{M}-c_{1} D_{1}^{*}\right) \otimes \Gamma_{1}\right] e(t)-e^{T}(t-h(t)) \\
& \cdot\left[\left(\frac{c_{2}}{2}-\Pi_{2}\right)\left(I_{N} \otimes \Gamma_{2}\right)\right] e(t-h(t))-\int_{t-k(t)}^{t} e^{T}(\theta) \\
& \cdot\left[\left(\frac{c_{3} k}{2}-\Pi_{3}\right)\left(I_{N} \otimes \Gamma_{3}\right)\right] e(\theta) d \theta
\end{aligned}
$$

where $\mathscr{M}=\Pi_{1} I_{N}+c_{1} G_{1}$. Note that the matrix $\mathscr{M}$ is symmetric. Let

$$
\mathscr{M}-D_{1}^{*}=\left[\begin{array}{cc}
\widetilde{M}_{1}-\widetilde{D}_{1 l}^{*} & \widetilde{\mathscr{M}}_{3} \\
\widetilde{M}_{3}^{T} & \widetilde{\mathscr{M}}_{2}
\end{array}\right]
$$

where $\widetilde{\mathscr{M}}_{2}$ is the minor matrix of $\mathscr{M}$ by removing its first $l(1 \leq l<N)$ row-column pairs, $\widetilde{M}_{1}$ and $\widetilde{M}_{3}$ are matrices with appropriate dimensions, and $\widetilde{D}_{1 l}^{*}=\operatorname{diag}\left(d_{11}^{*}\right.$, $\left.d_{12}^{*}, \ldots, d_{1 l}^{*}\right)$. If $\lambda_{\max }\left(\left(G_{1}+G_{1}^{T}\right) / 2\right)_{l}<-\Pi_{1} / c_{1}$ and with Lemma 9, we have $\widetilde{\mathscr{M}}_{2}<0$. Therefore, one can choose suitable positive constants $d_{1 i}^{*}>0, i=1,2, \ldots, l$, such that $d_{1 i}^{*}>\lambda_{\text {max }}\left(\widetilde{\mathscr{M}}_{1}-\widetilde{\mathscr{M}}_{3} \widetilde{\mathscr{M}}_{2}^{-1} \widetilde{\mathscr{M}}_{3}^{T}\right)$. It follows from Lemma 11 and $\widetilde{M}_{2}<0$ that $\mathscr{M}-c_{1} D_{1}^{*}<0$. Then, by $\Gamma_{1}>0$ and (29), we can conclude that

$$
\dot{V}(t) \leq-e^{T}(t-h(t))\left(\left(\frac{c_{2}}{2}-\Pi_{2}\right)\left(I_{N} \otimes \Gamma_{2}\right)\right)
$$




$$
\begin{gathered}
\cdot e(t-h(t))-\left(\int_{t-k(t)}^{t} e(\theta) d \theta\right)^{T} \\
\cdot\left(\left(\frac{c_{3} k}{2}-\Pi_{3}\right)\left(I_{N} \otimes \Gamma_{3}\right)\right) \\
\cdot\left(\int_{t-k(\mathrm{t})}^{t} e(\theta) d \theta\right) .
\end{gathered}
$$

We only need to choose the suitable positive constants $\bar{d}_{2}^{*}$ and $\bar{d}_{3}^{*}$ such that

$$
\begin{aligned}
\bar{d}_{2}^{*} & -\frac{1}{c_{2} \varepsilon_{4}}\left[\varepsilon_{2} \lambda_{\max }\left(I_{N} \otimes F_{2}^{T} F_{2}\right)+c_{2} \varepsilon_{7}\right. \\
& \left.+c_{2} \varepsilon_{8} \lambda_{\max }\left(I_{N} \otimes E_{2}^{T} E_{2}\right)-\lambda_{\min }\left(I_{N} \otimes \Gamma_{2}\right)\right]>0, \\
\bar{d}_{3}^{*} & -\frac{1}{c_{3} \varepsilon_{5}}\left[\varepsilon_{3} \lambda_{\max }\left(I_{N} \otimes F_{3}^{T} F_{3}\right)+c_{3} \varepsilon_{9}\right. \\
& \left.+c_{3} \varepsilon_{10} \lambda_{\max }\left(I_{N} \otimes E_{3}^{T} E_{3}\right)-\lambda_{\min }\left(I_{N} \otimes \Gamma_{3}\right)\right]>0 .
\end{aligned}
$$

We can choose $\bar{d}_{2}^{*}$ and $\bar{d}_{3}^{*}$ satisfying (32) and (33), respectively. Since $\Gamma_{1}, \Gamma_{2}$, and $\Gamma_{3}$ are positive definite matrix, we know that $\dot{V}(t) \leq 0$. Therefore, pinning controlled delayed neural networks (1) globally asymptotically synchronize to trajectory (6) if conditions (20), (21), and (22) hold. Then the controlled neural networks (1) are function projective synchronization.

Remark 13. If there are no uncertain parameters in coupled delayed neural networks (1), $\Delta \Gamma_{1}=\Delta \Gamma_{2}=\Delta \Gamma_{3}=0$. One can obtain similar synchronization results.

Remark 14. The nodes pinned for directed networks are chosen as follows.

Step 1. Choose some appropriate parameters $\varepsilon_{i}, i=1,2$, $\ldots, 10$, by taking appropriate $\bar{d}_{1 i}^{*}, i=1,2, \ldots, l, \bar{d}_{2}^{*}$, and $\bar{d}_{3}^{*}$ such that the conditions in Theorem 12 are feasible.

Step 2. The $l$ pinned nodes are sorted according to the pinned-node selection scheme studied [26], for the pinning controlled error neural network (17); so, the nodes to be pinned are chosen in the particular order. Let $l=1$, if the first inequalities of Theorem 12 are satisfied, and then the least number is 1 ; otherwise, go to the next step.

Step 3. If condition (20) is not satisfied, increase $l(l=l+1)$ gradually with more network nodes to the pinned node based on the order in Step 2 particularly until condition (20) holds.

For undirected networks, for example, the small-world network [31], the scale-free network [32], and the WattsStrogatz network [39], we can randomly choose a set of pinned nodes to satisfy condition (20) by increasing the number of pinned nodes $l$.
3.2. FPS under Hybrid Adaptive Control. The nonlinear and adaptive controls are designed to realize FPS of neural networks with mixed time-varying delays and uncertainties asymmetric coupling. Then we have the following controlled form:

$$
\mathcal{U}_{i}(t)=u_{i 1}(t)+u_{i 2}(t), \quad i=1,2, \ldots, N
$$

where

$$
\begin{aligned}
& u_{i 1}(t)=\dot{\alpha}(t) s(t)-W_{1}\left[\alpha(t) f_{1}(s(t))\right. \\
& \left.+f_{1}(\alpha(t) s(t))\right]-W_{2}\left[\alpha(t) f_{2}(s(t-h(t)))\right. \\
& \left.+f_{2}(\alpha(t) s(t-h(t)))\right] \\
& -W_{3} \int_{t-k(t)}^{t}\left[\alpha(t) f_{3}(s(\theta))-f_{3}(\alpha(t) s(\theta))\right] d \theta, \\
& \quad i=1,2, \ldots, N, \\
& u_{i 2}(t)=-c_{1} d_{1 i}(t) \Gamma_{1} e_{i}(t)-c_{2} d_{2 i}(t) \Gamma_{2} e_{i}(t-h(t)) \\
& -c_{3} d_{3 i}(t) \Gamma_{3} \int_{t-k(t)}^{t} e_{i}(\theta) d \theta, \quad i=1,2, \ldots, N .
\end{aligned}
$$

Then, substituting (34) into (8), we have

$$
\begin{aligned}
\dot{e}_{i}(t)= & -A e_{i}(t)+W_{1} \tilde{f}_{1}\left(e_{i}(t)\right) \\
& +W_{2} \tilde{f}_{2}\left(e_{i}(t-h(t))\right) \\
& +W_{3} \int_{t-k(t)}^{t} \tilde{f}_{3}\left(e_{i}(\theta)\right) d \theta \\
& +c_{1} \sum_{j=1}^{N} G_{1_{i j}}\left(\Gamma_{1}+\Delta \Gamma_{1}\right) e_{j}(t) \\
& +c_{2} \sum_{j=1}^{N} G_{2_{i j}}\left(\Gamma_{2}+\Delta \Gamma_{2}\right) e_{j}(t-h(t)) \\
& +c_{3} \sum_{j=1}^{N} G_{3_{i j}}\left(\Gamma_{3}+\Delta \Gamma_{3}\right) \int_{t-k(t)}^{t} e_{j}(\theta) d \theta \\
& -c_{1} d_{1 i}(t) \Gamma_{1} e_{i}(t) \\
& -c_{2} d_{2 i}(t) \Gamma_{2} e_{i}(t-h(t)) \\
& -c_{3} d_{3 i}(t) \Gamma_{3} \int_{t-k(t)}^{t} e_{i}(\theta) d \theta,
\end{aligned}
$$$$
i=1,2, \ldots, N,
$$$$
\dot{d}_{1 i}(t)=\beta_{i 1} e_{i}^{T}(t) \Gamma_{1} e_{i}(t), \quad i=1,2, \ldots, N,
$$$$
\dot{d}_{2 i}(t)=\beta_{i 2} e_{i}^{T}(t) \Gamma_{2} e_{i}(t-h(t)), \quad i=1,2, \ldots, N,
$$$$
\dot{d}_{3 i}(t)=\beta_{i 3} e_{i}^{T}(t) \Gamma_{3} \int_{t-k(t)}^{t} e_{i}(\theta) d \theta, \quad i=1,2, \ldots, N,
$$ 
where

$$
\begin{aligned}
& \Omega_{1}=\frac{1}{\lambda_{\min }\left(I_{N} \otimes \Gamma_{1}\right)}\left[-\lambda_{\max }\left(I_{N} \otimes A\right)\right. \\
& +\frac{1}{2 \varepsilon_{1}} \lambda_{\max }\left(I_{N} \otimes W_{1} W_{1}^{T}\right)+\frac{\varepsilon_{1}}{2} \lambda_{\max }\left(I_{N} \otimes F_{1}^{T} F_{1}\right) \\
& +c_{1} \lambda_{\max }\left(G_{1}\right) \lambda_{\max }\left(\Gamma_{1}\right)+\frac{c_{3} k^{2}}{2} \lambda_{\max }\left(I_{N} \otimes \Gamma_{3}\right) \\
& +\frac{1}{2 \varepsilon_{2}} \lambda_{\max }\left(I_{N} \otimes W_{2} W_{2}^{T}\right) \\
& +\frac{1}{2 \varepsilon_{3}} \lambda_{\max }\left(I_{N} \otimes W_{3} W_{3}^{T}\right) \\
& +\frac{c_{1} \varepsilon_{6}}{2} \lambda_{\max }\left(I_{N} \otimes E_{1}^{T} E_{1}\right) \\
& +\frac{c_{1}}{2 \varepsilon_{6}} \lambda_{\max }\left(G_{1} G_{1}^{T}\right) \lambda_{\max }\left(M M^{T}\right) \\
& +\frac{c_{2}}{2 \varepsilon_{7}} \lambda_{\max }\left(G_{2} G_{2}^{T}\right) \lambda_{\max }\left(\Gamma_{2} \Gamma_{2}^{T}\right) \\
& +\frac{\mathcal{c}_{2}}{2 \varepsilon_{8}} \lambda_{\max }\left(G_{2} G_{2}^{T}\right) \lambda_{\max }\left(M M^{T}\right) \\
& +\frac{c_{3}}{2 \varepsilon_{9}} \lambda_{\max }\left(G_{3} G_{3}^{T}\right) \lambda_{\max }\left(\Gamma_{3} \Gamma_{3}^{T}\right) \\
& +\frac{\mathcal{C}_{3}}{2 \varepsilon_{10}} \lambda_{\max }\left(G_{3} G_{3}^{T}\right) \lambda_{\max }\left(M M^{T}\right) \\
& +\frac{c_{2}}{2(1-\mu)} \lambda_{\max }\left(I_{N} \otimes \Gamma_{2}\right) \\
& +\frac{c_{2} d_{2}^{*}}{2 \varepsilon_{4}} \lambda_{\max }\left(I_{N} \otimes \Gamma_{2} \Gamma_{2}^{T}\right) \\
& \left.+\frac{c_{3} d_{3}^{*}}{2 \varepsilon_{5}} \lambda_{\max }\left(I_{N} \otimes \Gamma_{3} \Gamma_{3}^{T}\right)\right] \text {, } \\
& \Omega_{2}=\frac{1}{2 \lambda_{\min }\left(I_{N} \otimes \Gamma_{2}\right)}\left[\varepsilon_{2} \lambda_{\text {max }}\left(I_{N} \otimes F_{2}^{T} F_{2}\right)+c_{2} \varepsilon_{7}\right. \\
& \left.+c_{2} \varepsilon_{8} \lambda_{\max }\left(I_{N} \otimes E_{2}^{T} E_{2}\right)+c_{2} d_{2}^{*} \varepsilon_{4}\right] \text {, } \\
& \Omega_{3}=\frac{1}{2 \lambda_{\text {min }}\left(I_{N} \otimes \Gamma_{3}\right)}\left[\varepsilon_{3} \lambda_{\max }\left(I_{N} \otimes F_{3}^{T} F_{3}\right)+c_{3} \varepsilon_{9}\right. \\
& \left.+c_{3} \varepsilon_{10} \lambda_{\max }\left(I_{N} \otimes E_{3}^{T} E_{3}\right)+c_{3} d_{3}^{*} \varepsilon_{5}\right] \text {, } \\
& \xi(t)=\left(e^{T}(t), e^{T}(t-h(t)),\left(\int_{t-k(t)}^{t} e(\theta) d \theta\right)^{T}\right)^{T} .
\end{aligned}
$$

By using adaptive controlling method, we get the following theorem.

Theorem 15. For some given synchronization scaling function $\alpha(t)$, neural networks (1) satisfying Assumptions 1, 2, and 3, and target system can realize function projective synchronization by the adaptive control law as shown in (34) if there exist positive constants $\varepsilon_{i}, i=1,2, \ldots, 10$, and by taking appropriate $d_{1}^{*}, d_{2}^{*}$, and $d_{3}^{*}$ such that

$$
\begin{aligned}
d_{1}^{*} & -\frac{\Omega_{1}}{c_{1}}>0, \\
d_{2}^{*} & -\frac{1}{c_{2} \varepsilon_{4}}\left[\varepsilon_{2} \lambda_{\max }\left(I_{N} \otimes F_{2}^{T} F_{2}\right)+c_{2} \varepsilon_{7}\right. \\
& \left.+c_{2} \varepsilon_{8} \lambda_{\max }\left(I_{N} \otimes E_{2}^{T} E_{2}\right)-\lambda_{\text {min }}\left(I_{N} \otimes \Gamma_{2}\right)\right]>0, \\
d_{3}^{*} & -\frac{1}{c_{3} \varepsilon_{5}}\left[\varepsilon_{3} \lambda_{\max }\left(I_{N} \otimes F_{3}^{T} F_{3}\right)+c_{3} \varepsilon_{9}\right. \\
& \left.+c_{3} \varepsilon_{10} \lambda_{\max }\left(I_{N} \otimes E_{3}^{T} E_{3}\right)-\lambda_{\min }\left(I_{N} \otimes \Gamma_{3}\right)\right]>0 .
\end{aligned}
$$

Then controlled neural networks (1) are function projective synchronization.

Proof. Construct the following Lyapunov-Krasovskii functional candidate:

$$
V(t)=V_{1}(t)+V_{2}(t)+V_{3}(t)+V_{4}(t),
$$

where

$$
\begin{aligned}
V_{1}(t)= & \frac{1}{2} \sum_{i=1}^{N} e_{i}^{T}(t) e_{i}(t), \\
V_{2}(t)= & \frac{c_{2}}{2(1-\mu)} \sum_{i=1}^{N} \int_{t-h(t)}^{t} e_{i}^{T}(\theta) \Gamma_{2} e_{i}(\theta) d \theta, \\
V_{3}(t)= & \frac{c_{3} k}{2} \sum_{i=1}^{N} \int_{-k}^{0} \int_{t+s}^{t} e_{i}^{T}(\theta) \Gamma_{3} e_{i}(\theta) d \theta d s, \\
V_{4}(t)= & \frac{c_{1}}{2} \sum_{i=1}^{N} \frac{1}{\beta_{i 1}}\left(d_{1 i}(t)-d_{1}^{*}\right)^{2} \\
& +\frac{c_{2}}{2} \sum_{i=1}^{N} \frac{1}{\beta_{i 2}}\left(d_{2}(t)-d_{2 i}^{*}\right)^{2} \\
& +\frac{c_{3}}{2} \sum_{i=1}^{N} \frac{1}{\beta_{i 3}}\left(d_{3}(t)-d_{3 i}^{*}\right)^{2} .
\end{aligned}
$$

By taking the derivative of $V(t)$ along the trajectories of system (36) that is similar to the proof of Theorem 12, we obtain

$$
\begin{aligned}
\dot{V}(t) & \leq e^{T}(t)\left[-\left(I_{N} \otimes A\right)+\frac{1}{2 \varepsilon_{1}}\left(I_{N} \otimes W_{1} W_{1}^{T}\right)\right. \\
+ & \frac{\varepsilon_{1}}{2}\left(I_{N} \otimes F_{1}^{T} F_{1}\right)+\frac{c_{2}}{2(1-\mu)}\left(I_{N} \otimes \Gamma_{2}\right) \\
& -c_{1} d_{1}^{*}\left(I_{N} \otimes \Gamma_{1}\right)+c_{1}\left(G_{1} \otimes \Gamma_{1}\right)+\frac{c_{3} k^{2}}{2}\left(I_{N} \otimes \Gamma_{3}\right) \\
& +\frac{1}{2 \varepsilon_{2}}\left(I_{N} \otimes W_{2} W_{2}^{T}\right)+\frac{1}{2 \varepsilon_{3}}\left(I_{N} \otimes W_{3} W_{3}^{T}\right)
\end{aligned}
$$




$$
\begin{aligned}
& +\frac{c_{1}}{2 \varepsilon_{6}}\left(G_{1} G_{1}^{T} \otimes M M^{T}\right)+\frac{c_{1} \varepsilon_{6}}{2}\left(I_{N} \otimes E_{1}^{T} E_{1}\right) \\
& +\frac{c_{2}}{2 \varepsilon_{7}}\left(G_{2} G_{2}^{T} \otimes \Gamma_{2} \Gamma_{2}^{T}\right)+\frac{c_{2}}{2 \varepsilon_{8}}\left(G_{2} G_{2}^{T} \otimes M M^{T}\right) \\
& +\frac{c_{3}}{2 \varepsilon_{9}}\left(G_{3} G_{3}^{T} \otimes \Gamma_{3} \Gamma_{3}^{T}\right)+\frac{c_{3}}{2 \varepsilon_{10}}\left(G_{3} G_{3}^{T} \otimes M M^{T}\right) \\
& \left.+\frac{c_{2} d_{2}^{*}}{2 \varepsilon_{4}}\left(I_{N} \otimes \Gamma_{2} \Gamma_{2}^{T}\right)+\frac{c_{3} d_{3}^{*}}{2 \varepsilon_{5}}\left(I_{N} \otimes \Gamma_{3} \Gamma_{3}^{T}\right)\right] e(t) \\
& -e^{T}(t-h(t))\left[\frac{c_{2}}{2}\left(I_{N} \otimes \Gamma_{2}\right)-\Omega_{2}\left(I_{N} \otimes \Gamma_{2}\right)\right] e(t \\
& -h(t))-\int_{t-k(t)}^{t} e^{T}(\theta) \\
& \cdot\left[\frac{c_{3} k}{2}\left(I_{N} \otimes \Gamma_{3}\right)-\Omega_{3}\left(I_{N} \otimes \Gamma_{3}\right)\right] e(\theta) d \theta \\
& \leq e^{T}(t)\left[\left(\Omega_{1}-c_{1} d_{1}^{*}\right)\left(I_{N} \otimes \Gamma_{1}\right)\right] e(t)-e^{T}(t-h(t)) \\
& \cdot\left[\left(\frac{c_{2}}{2}-\Omega_{2}\right)\left(I_{N} \otimes \Gamma_{2}\right)\right] e(t-h(t)) \\
& -\left(\int_{t-k(t)}^{t} e(\theta) d \theta\right)^{T}\left[\left(\frac{c_{3} k}{2}-\Omega_{3}\right)\left(I_{N} \otimes \Gamma_{3}\right)\right] \\
& \cdot\left(\int_{t-k(t)}^{t} e(\theta) d \theta\right) .
\end{aligned}
$$

It is obvious that there exist sufficiently large positive constants $d_{1}^{*}, d_{2}^{*}$, and $d_{3}^{*}$ such that

$$
\begin{aligned}
d_{1}^{*} & -\frac{\Omega_{1}}{c_{1}}>0, \\
d_{2}^{*} & -\frac{1}{c_{2} \varepsilon_{4}}\left[\varepsilon_{2} \lambda_{\max }\left(I_{N} \otimes F_{2}^{T} F_{2}\right)+c_{2} \varepsilon_{7}\right. \\
& \left.+c_{2} \varepsilon_{8} \lambda_{\max }\left(I_{N} \otimes E_{2}^{T} E_{2}\right)-\lambda_{\min }\left(I_{N} \otimes \Gamma_{2}\right)\right]>0, \\
d_{3}^{*} & -\frac{1}{c_{3} \varepsilon_{5}}\left[\varepsilon_{3} \lambda_{\max }\left(I_{N} \otimes F_{3}^{T} F_{3}\right)+c_{3} \varepsilon_{9}\right. \\
& \left.+c_{3} \varepsilon_{10} \lambda_{\max }\left(I_{N} \otimes E_{3}^{T} E_{3}\right)-\lambda_{\min }\left(I_{N} \otimes \Gamma_{3}\right)\right]>0 .
\end{aligned}
$$

We can choose $d_{1}^{*}, d_{2}^{*}$, and $d_{3}^{*}$ satisfying (42), (43), and (44), respectively. The remaining proof is similar to Theorem 12 and omitted.

\section{Numerical Simulations}

In this section, we provide several numerical examples to demonstrate the feasibility of the proposed method.
Example 16. Consider a two-dimensional neural network with time-varying delay presented in the following system:

$$
\begin{aligned}
\dot{s}(t)= & -A s(t)+W_{1} f_{1}(s(t))+W_{2} f_{2}(s(t-h(t))) \\
& +W_{3} \int_{t-k(t)}^{t} f_{3}(s(\theta)) d \theta,
\end{aligned}
$$

where $s(t)=\left[\begin{array}{ll}s_{1}(t) & s_{2}(t)\end{array}\right]^{T} \in \mathscr{R}^{2}$ is the state vector of the network, the activation function $f_{j}\left(x_{i}(t)\right)=\tanh \left(x_{i}(t)\right),(i=$ $1,2, j=1,2,3)$, the delays $h(t)=1-0.9 \sin (t)$ and $k(t)=$ $0.2 \sin (t)$, and the other matrices are as follows:

$$
\begin{aligned}
A & =\left[\begin{array}{ll}
1 & 0 \\
0 & 1
\end{array}\right], \\
W_{1} & =\left[\begin{array}{cc}
2 & -0.1 \\
-5 & 4.5
\end{array}\right], \\
W_{2} & =\left[\begin{array}{cc}
-1.5 & -0.1 \\
-0.2 & -4
\end{array}\right], \\
W_{3} & =\left[\begin{array}{cc}
-0.3 & 0.1 \\
0.1 & -0.2
\end{array}\right] .
\end{aligned}
$$

The dynamical chaotic trajectory of neural network (45) with initial conditions $s_{1}(r)=0.6$ and $s_{2}(r)=0.5, \forall r \in[-2,0]$, is shown in Figure 1.

Afterwards, the FPS problems for the nonlinear and adaptive pinning controlled network consisting of $N$ twodimensional neural networks are described as follows:

$$
\begin{aligned}
\dot{x}_{i}(t)= & -A x_{i}(t)+W_{1} f_{1}\left(x_{i}(t)\right) \\
& +W_{2} f_{2}\left(x_{i}(t-h(t))\right) \\
& +W_{3} \int_{t-k(t)}^{t} f_{3}\left(x_{i}(\theta)\right) d \theta \\
& +c_{1} \sum_{j=1}^{N} G_{1_{i j}}\left(\Gamma_{1}+\Delta \Gamma_{1}\right) x_{j}(t) \\
& +c_{2} \sum_{j=1}^{N} G_{2_{i j}}\left(\Gamma_{2}+\Delta \Gamma_{2}\right) x_{j}(t-h(t)) \\
& +c_{3} \sum_{j=1}^{N} G_{3_{i j}}\left(\Gamma_{3}+\Delta \Gamma_{3}\right) \int_{t-k(t)}^{t} x_{j}(\theta) d \theta \\
& +\mathcal{U}_{i}(t), \quad i=1,2, \ldots, N,
\end{aligned}
$$




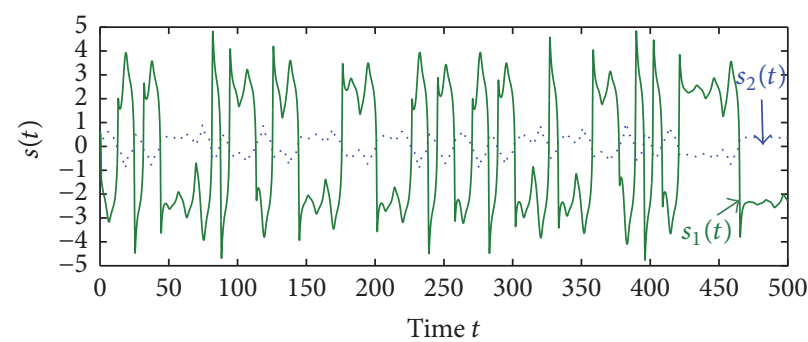

(a)

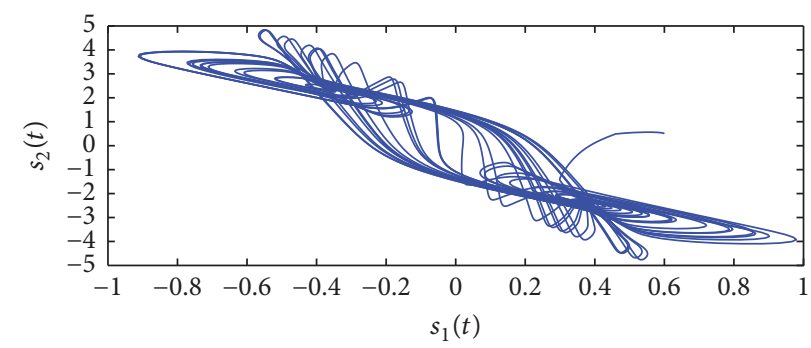

(b)

FIGURE 1: (a) Chaotic trajectory of neural network (45). (b) Phase portrait of the strange attractor.
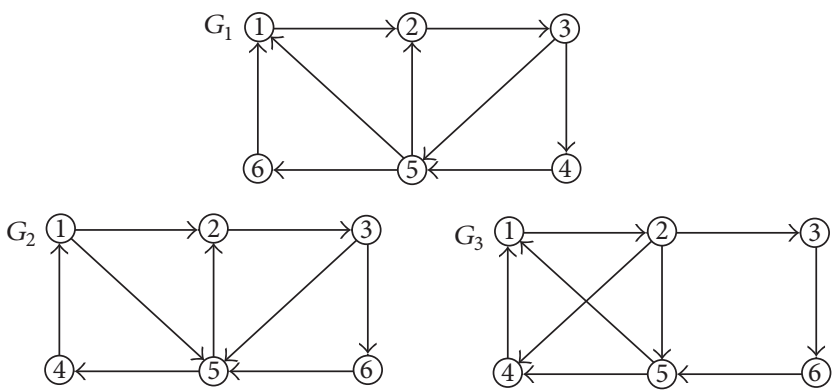

Figure 2: Simple directed neural network with 6 nodes.

where $x_{i}(t)=\left[x_{i 1}(t), x_{i 2}(t)\right]^{T} \in \mathscr{R}^{2}$ is the state variable of the $i$ th node. The inner-coupling matrices with uncertainties are

$$
\begin{aligned}
\Gamma_{1} & =\left[\begin{array}{ll}
5 & 0 \\
0 & 5
\end{array}\right], \\
\Gamma_{2} & =\left[\begin{array}{ll}
2 & 0 \\
0 & 2
\end{array}\right], \\
\Gamma_{3} & =\left[\begin{array}{ll}
1 & 0 \\
0 & 1
\end{array}\right], \\
M & =\left[\begin{array}{ll}
0.2 & 0 \\
0 & 0.2
\end{array}\right], \\
\nabla(t) & =\left[\begin{array}{cc}
\sin (t) & 0 \\
0 & \cos (t)
\end{array}\right], \\
E_{r} & =\left[\begin{array}{cc}
0.1 & 0 \\
0 & 0.1
\end{array}\right] \quad(r=1,2,3),
\end{aligned}
$$

and the other parameters are the same as those in (45).

Directed Neural Network. We consider the directed neural networks as shown in Figure 2.

Choose the following parameters: the time-varying scaling function $\alpha(t)=1.3 \sin (2 \pi / 15)$, the coupling strength values $c_{1}=16, c_{2}=1$, and $c_{3}=1$, and the positive constants $\varepsilon_{1}=12.5, \varepsilon_{2}=4.5, \varepsilon_{3}=0.8, \varepsilon_{4}=0.7, \varepsilon_{5}=0.8, \varepsilon_{6}=7.2$, $\varepsilon_{7}=3.7, \varepsilon_{8}=5, \varepsilon_{9}=5$, and $\varepsilon_{10}=12$. According to Figure 2, the controller networks with 6 nodes are described with the outer-coupling matrices by

$$
\begin{aligned}
& G_{1}=\left[\begin{array}{cccccc}
-2 & 0 & 0 & 0 & 1 & 1 \\
1 & -2 & 0 & 0 & 1 & 0 \\
0 & 1 & -1 & 0 & 0 & 0 \\
0 & 0 & 1 & -1 & 0 & 0 \\
0 & 0 & 1 & 1 & -2 & 0 \\
0 & 0 & 0 & 0 & 1 & -1
\end{array}\right], \\
& G_{2}=\left[\begin{array}{cccccc}
-1 & 0 & 0 & 1 & 0 & 0 \\
1 & -2 & 0 & 0 & 1 & 0 \\
0 & 1 & -1 & 0 & 0 & 0 \\
0 & 0 & 0 & -1 & 1 & 0 \\
1 & 0 & 1 & 0 & -3 & 1 \\
0 & 0 & 1 & 0 & 0 & -1
\end{array}\right], \\
& G_{3}=\left[\begin{array}{cccccc}
-2 & 0 & 0 & 1 & 1 & 0 \\
1 & -1 & 0 & 0 & 0 & 0 \\
0 & 1 & -1 & 0 & 0 & 0 \\
0 & 1 & 0 & -2 & 1 & 0 \\
0 & 1 & 0 & 0 & -2 & 1 \\
0 & 0 & 1 & 0 & 0 & -1
\end{array}\right] .
\end{aligned}
$$

Solution. As presented in Figure 2, there are the out-degrees and the in-degree of nodes. According to the pinned-node selection scheme, we rearrange the nodes in $G_{1}$ and the new 


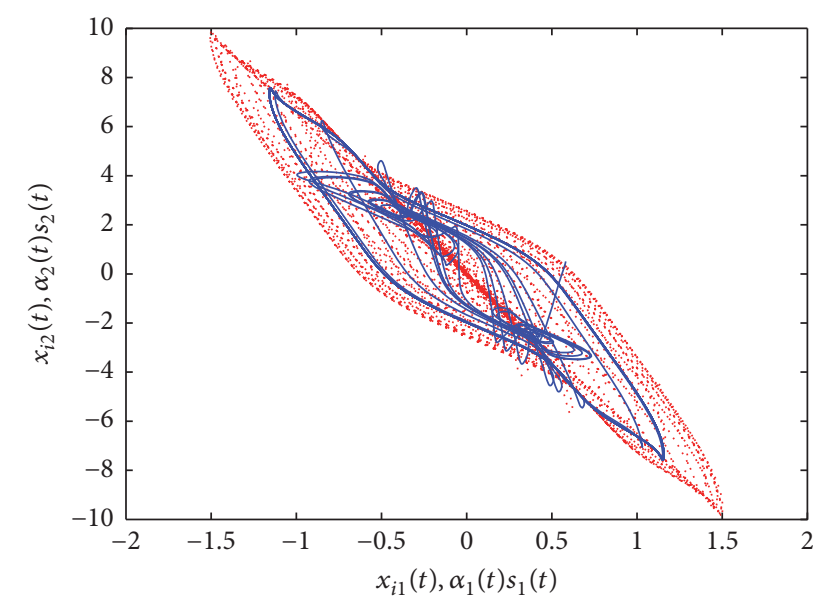

Drive system

_ Response network

FIGURE 3: Chaotic behavior of isolate node $\alpha(t) s(t)$ (45) and node $x_{i}(t)$ (47) with the time-varying scaling function $\alpha(t)$.

order is $3,5,4,6,1,2$. When node 3 is chosen as pinned node with $l=1$, we obtain $d_{1 l}=5, \bar{d}_{2}^{*}=2.0125$, and $\bar{d}_{3}^{*}=3.4560$. Thus, it is confirmed that pinning conditions (20)-(22) in Theorem 12 hold:

$$
\begin{aligned}
\lambda_{\max }\left(\frac{G_{1}+G_{1}^{T}}{2}\right)_{l} & =-0.2989<-\frac{\Pi_{1}}{c_{1}}=-\frac{4.5659}{15} \\
& =-0.2854 .
\end{aligned}
$$

Figure 3 shows the chaotic behavior of isolate node $\alpha(t) s(t)$ (45) and node $x_{i}(t)$ (47), with the time-varying scaling function $\alpha(t)$. Figure 4 shows the FPS errors between the states of isolate node $\alpha(t) s(t)$ (45) and node $x_{i}(t)$ (47), where $e_{i j}(t)=x_{i j}(t)-\alpha_{j}(t) s_{j}(t)$ for $i=1, \ldots, 6, j=$ 1,2 , without nonlinear and adaptive pinning control (14). Figure 5 shows the FPS errors between the states of isolate node $\alpha(t) s(t)$ (45) and node $x_{i}(t)$ (47) where $e_{i j}(t)=x_{i j}(t)$ $\alpha_{j}(t) s_{j}(t)$ for $i=1, \ldots, 6, j=1,2$ with nonlinear and adaptive pinning control (14). Figure 6 gives the evolution of adaptive pinning feedback gains $d_{11}(t), d_{21}(t)$, and $d_{31}(t)$.

Undirected Neural Network. We consider the FPS for a largescale undirected Watts-Strogatz network with 30 identical nodes of the isolated dynamic network with mixed timevarying delays which is given in (47). Choose the timevarying scaling function $\alpha(t)=1.3 \sin (2 \pi / 15)$ and the coupling strength values $c_{1}=12, c_{2}=1$, and $c_{3}=1$. For a Watts-Strogatz network here, we set the parameters $[N=30$, $K=2$, and $\rho=0.65]$, $[N=30, K=1$, and $\rho=1]$, and $[N=30, K=1$, and $\rho=0.8]$, respectively. Then, the coupling matrices $G_{1}, G_{2}$, and $G_{3}$ can be randomly generated by the Watts-Strogatz models and are shown in Figures 7-9, respectively.
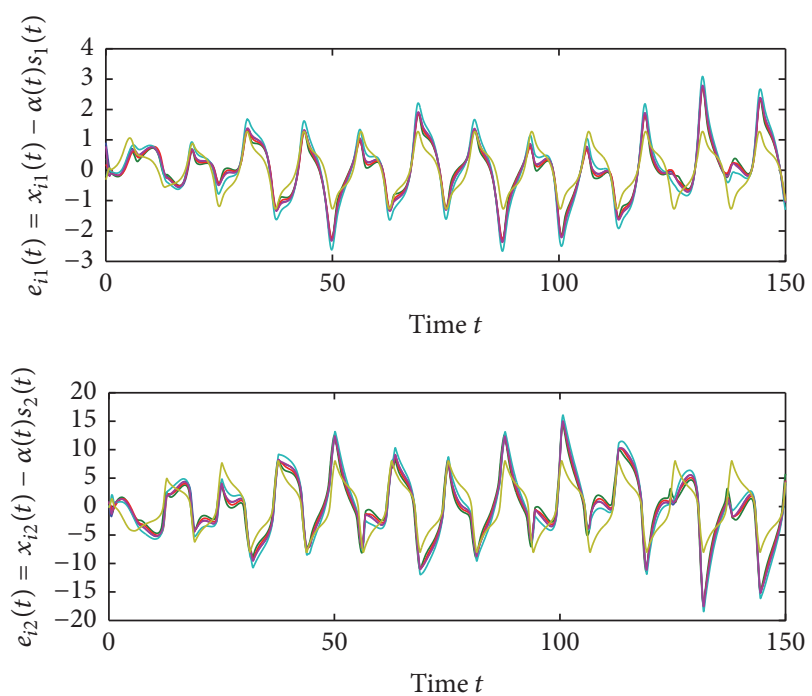

FIGURE 4: The FPS errors between the states of isolate node $\alpha(t) s(t)$ (45) and node $x_{i}(t)$ (47) without nonlinear and adaptive pinning control (14).
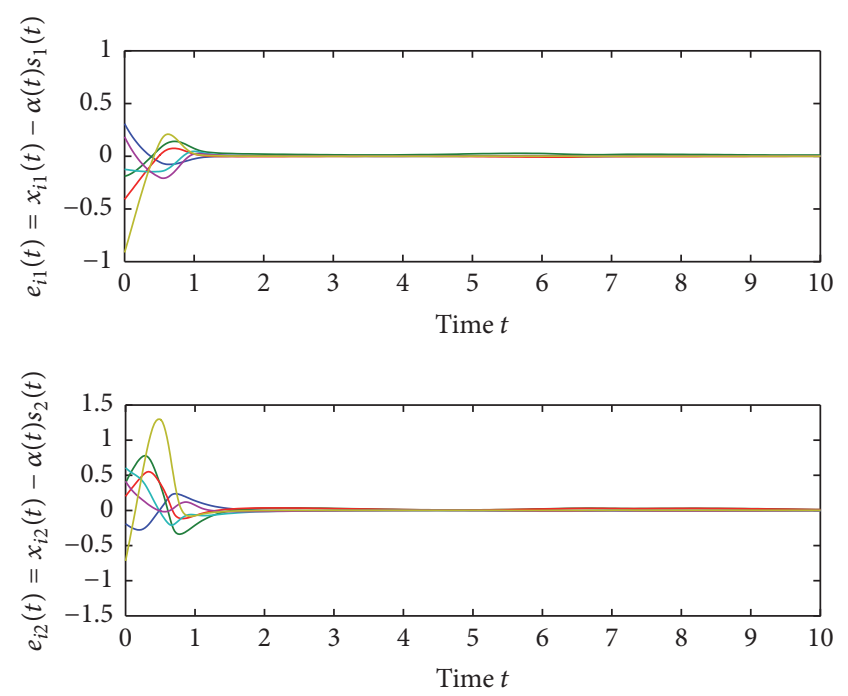

FIGURE 5: The FPS errors between the states of isolate node $\alpha(t) s(t)$ (45) and node $x_{i}(t)$ (47) with nonlinear and adaptive pinning control (14).

Solution. From conditions (20)-(22) in Theorem 12 and the positive constants $\varepsilon_{1}=12.5, \varepsilon_{2}=4.5, \varepsilon_{3}=0.8, \varepsilon_{4}=0.7$, $\varepsilon_{5}=0.8, \varepsilon_{6}=7.2, \varepsilon_{7}=3.7, \varepsilon_{8}=5, \varepsilon_{9}=5$, and $\varepsilon_{10}=12$, one can check that the last three conditions in Theorem 12 are satisfied. We study how to select pinned nodes of network. As $G_{1}$ is an undirected Watts-Strogatz network, the pinned nodes can be randomly chosen for the convenience of practical applications by randomly choosing ten network nodes; that is, $l=10$, and the feedback control gains are chosen as $d_{1 i}=30(i=1,2, \ldots, 10), \bar{d}_{2}^{*}=2.0125$, and $\bar{d}_{3}^{*}=3.4560$. From simple numerical calculation, it can 

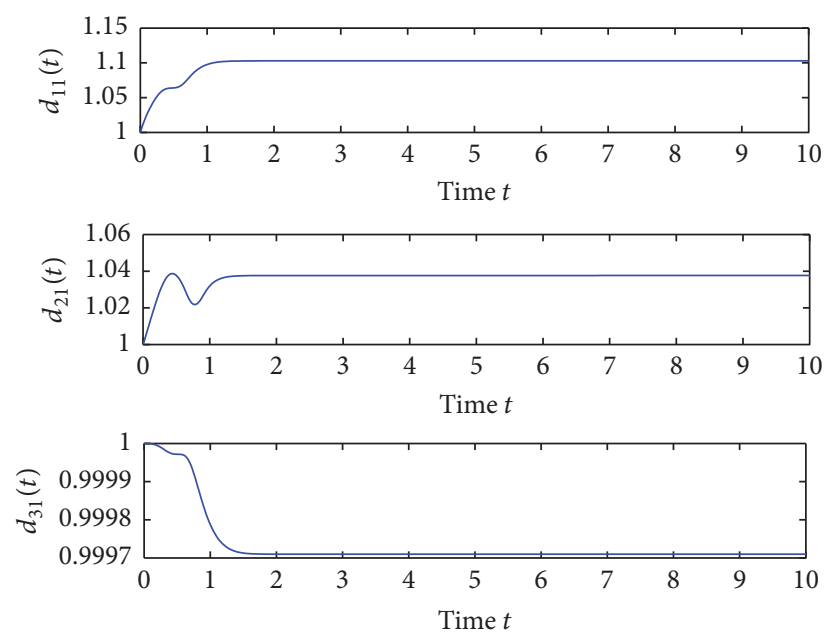

FIGURE 6: The evolution of adaptive pinning feedback gains $d_{11}(t)$, $d_{21}(t)$, and $d_{31}(t)$.

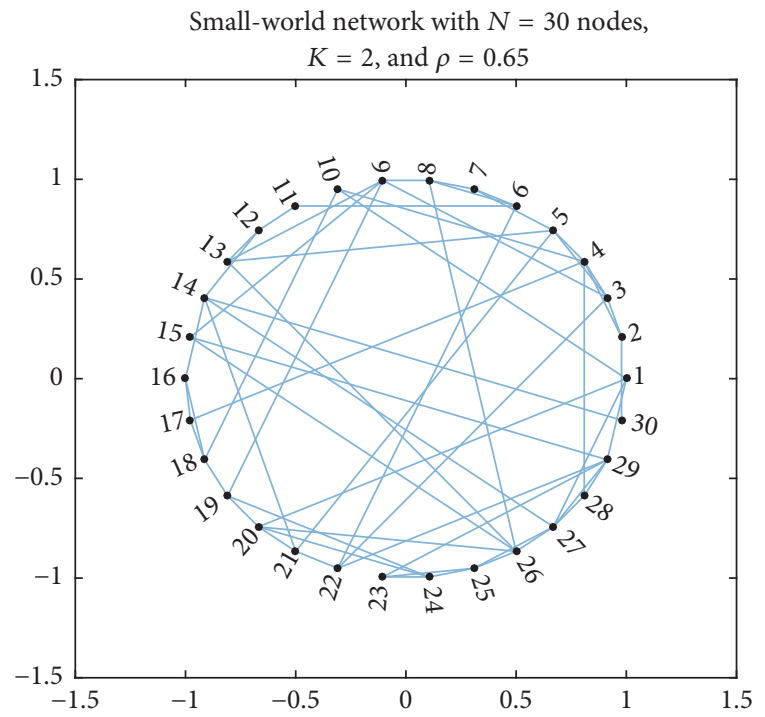

FIGURE 7: The topology structure of Watts-Strogatz neural network with $N=30, K=2$, and $\rho=0.65$.

be seen that pinning condition (20) holds:

$$
\begin{aligned}
\lambda_{\max }\left(\frac{G_{1}+G_{1}^{T}}{2}\right)_{l} & =-0.6407<-\frac{\Pi_{1}}{c_{1}}=-\frac{7.1110}{12} \\
& =-0.5926
\end{aligned}
$$

Figure 10 shows the FPS errors between the states of isolate node $\alpha(t) s(t)(45)$ and node $x_{i}(t)(47)$, where $e_{i j}(t)=$ $x_{i j}(t)-\alpha_{j}(t) s_{j}(t)$ for $i=1, \ldots, 6, j=1,2$, without nonlinear and adaptive pinning control (14). Figure 11 shows the FPS errors between the states of isolate node $\alpha(t) s(t)$ (45) and node $x_{i}(t)$ (47), where $e_{i j}(t)=x_{i j}(t)-\alpha_{j}(t) s_{j}(t)$ for $i=$ $1, \ldots, 6, j=1,2$, with nonlinear and adaptive pinning control (14). Figure 12 gives the evolution of adaptive pinning feedback gains $d_{1 i}(t), d_{2 i}(t)$, and $d_{3 i}(t)(i=1,2, \ldots, 10)$.

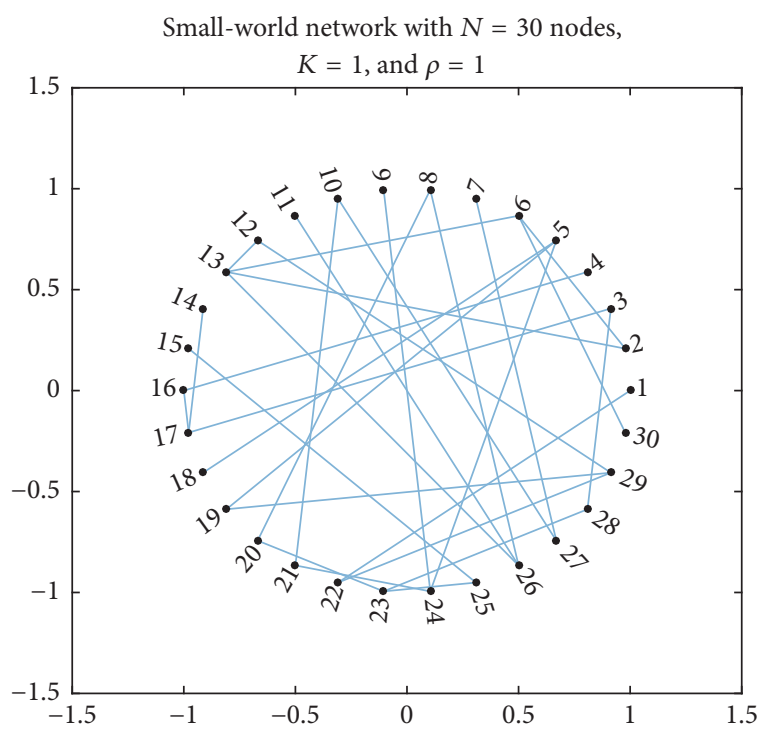

FIGURE 8: The topology structure of Watts-Strogatz neural network with $N=30, K=1$, and $\rho=1$.

Example 17. We consider the FPS problems for the nonlinear and adaptive controlled network consisting of $N$ two-dimensional neural networks (36). The directed neural networks are shown in Figure 13, where the constant coupling matrix is determined to be

$$
\begin{aligned}
& G_{1}=\left[\begin{array}{ccccc}
-1 & 0 & 0 & 0 & 1 \\
1 & -2 & 0 & 0 & 1 \\
0 & 1 & -1 & 0 & 0 \\
0 & 0 & 1 & -1 & 0 \\
0 & 0 & 1 & 1 & -2
\end{array}\right], \\
& G_{2}=\left[\begin{array}{ccccc}
-2 & 0 & 0 & 1 & 1 \\
1 & -1 & 0 & 0 & 0 \\
0 & 0 & -1 & 0 & 1 \\
0 & 1 & 1 & -2 & 0 \\
0 & 1 & 0 & 1 & -2
\end{array}\right], \\
& G_{3}=\left[\begin{array}{ccccc}
-1 & 0 & 0 & 1 & 0 \\
1 & -1 & 0 & 0 & 0 \\
1 & 1 & -2 & 0 & 0 \\
0 & 0 & 1 & -2 & 1 \\
0 & 1 & 0 & 0 & -1
\end{array}\right] .
\end{aligned}
$$

The inner-coupling matrices with uncertainties are the same as in (47), and the other parameters are the same as those in (45). Choose the time-varying scaling function $\alpha(t)=1.3 \sin (2 \pi / 15)$ and the coupling strength values $c_{1}=$ $2.5, c_{2}=0.2$, and $c_{3}=0.5$.

Solution. From conditions (38) in Theorem 15 and the positive constants $\varepsilon_{1}=0.1, \varepsilon_{2}=10.5, \varepsilon_{3}=3.5, \varepsilon_{4}=0.5, \varepsilon_{5}=0.7, \varepsilon_{6}=$ 


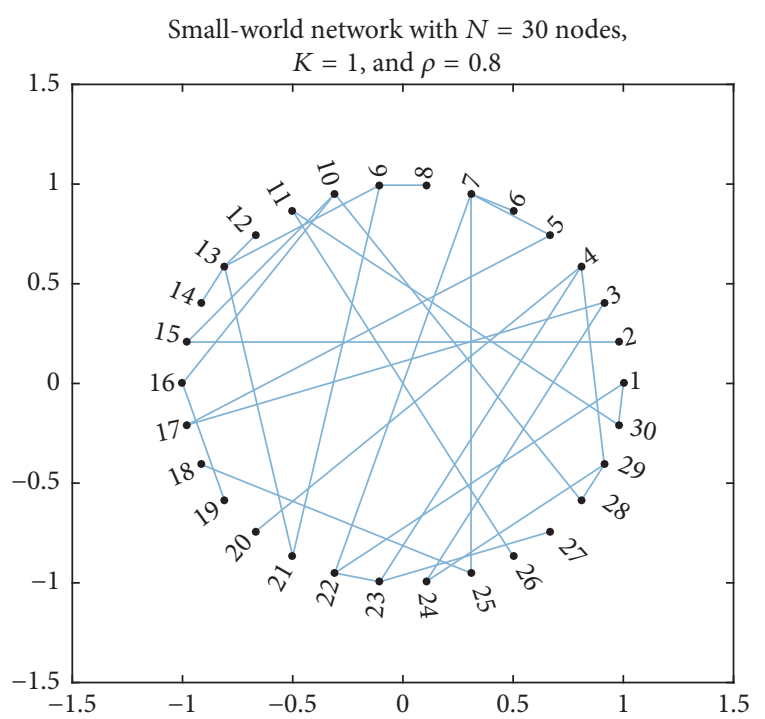

FIgURE 9: The topology structure of Watts-Strogatz neural network with $N=30, K=1$, and $\rho=0.8$.
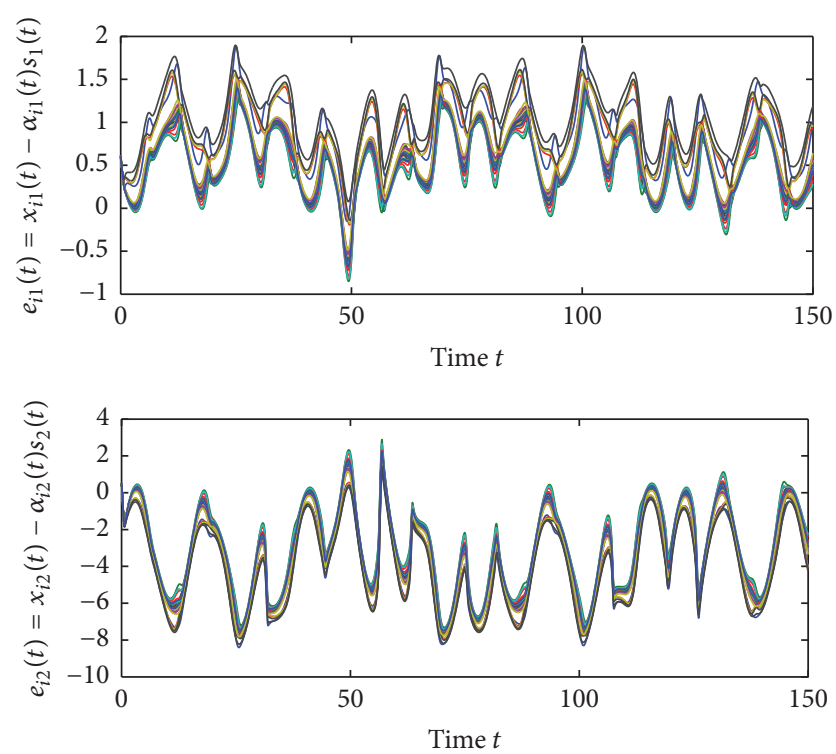

FIGURE 10: The FPS errors between the states of isolate node $\alpha(t) s(t)$ (45) and node $x_{i}(t)(47)$ without nonlinear and adaptive pinning control (14).

$10, \varepsilon_{7}=3.7, \varepsilon_{8}=6, \varepsilon_{9}=4.2$, and $\varepsilon_{10}=5$, one can check that the last three conditions in Theorem 15 are satisfied. Then, we obtain $d_{1}^{*}>3.4425, d_{2}^{*}>2.8000$, and $d_{3}^{*}>5.5199$.

Figure 14 shows the FPS errors between the states of isolate node $\alpha(t) s(t)$ of (45) and node $x_{i}(t)$ of (47), where $e_{i j}(t)=x_{i j}(t)-\alpha_{j}(t) s_{j}(t)$ for $i=1,2, j=1,2,3$, without nonlinear and adaptive control (34). Figure 15 shows the FPS errors between the states of isolate node $\alpha(t) s(t)$ of (45) and node $x_{i}(t)$ of $(47)$, where $e_{i j}(t)=x_{i j}(t)-\alpha_{j}(t) s_{j}(t)$ for $i=$ $1,2, j=1,2,3$, with nonlinear and adaptive control (34). Figure 16 gives the evolution of adaptive feedback gains $d_{11}(t)$, $d_{21}(t)$, and $d_{31}(t)$.
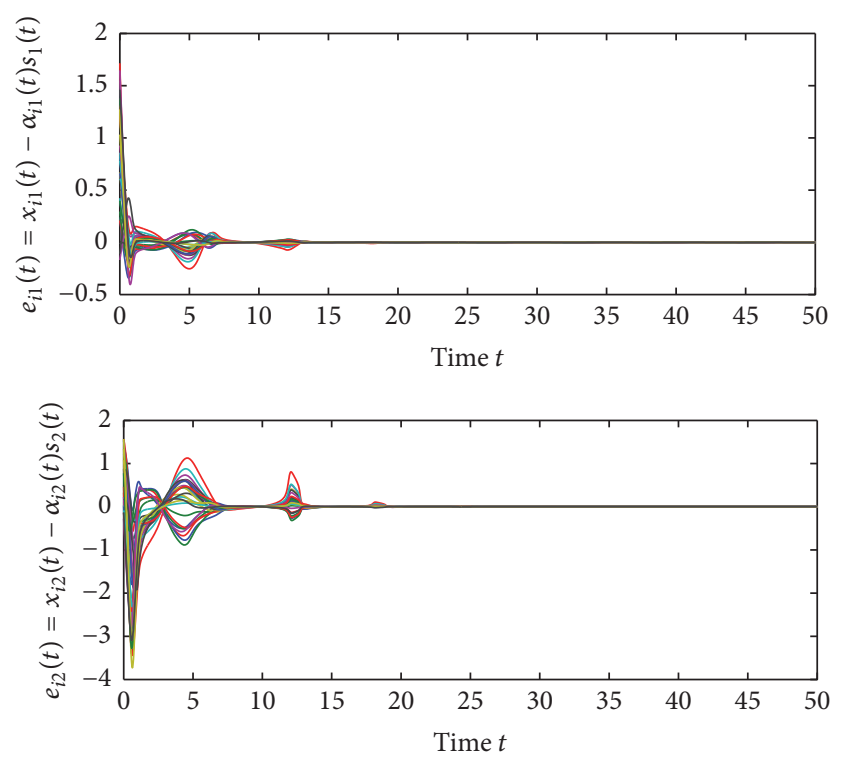

FIGURE 11: The FPS errors between the states of isolate node $\alpha(t) s(t)$ (45) and node $x_{i}(t)(47)$ with nonlinear and adaptive pinning control (14).
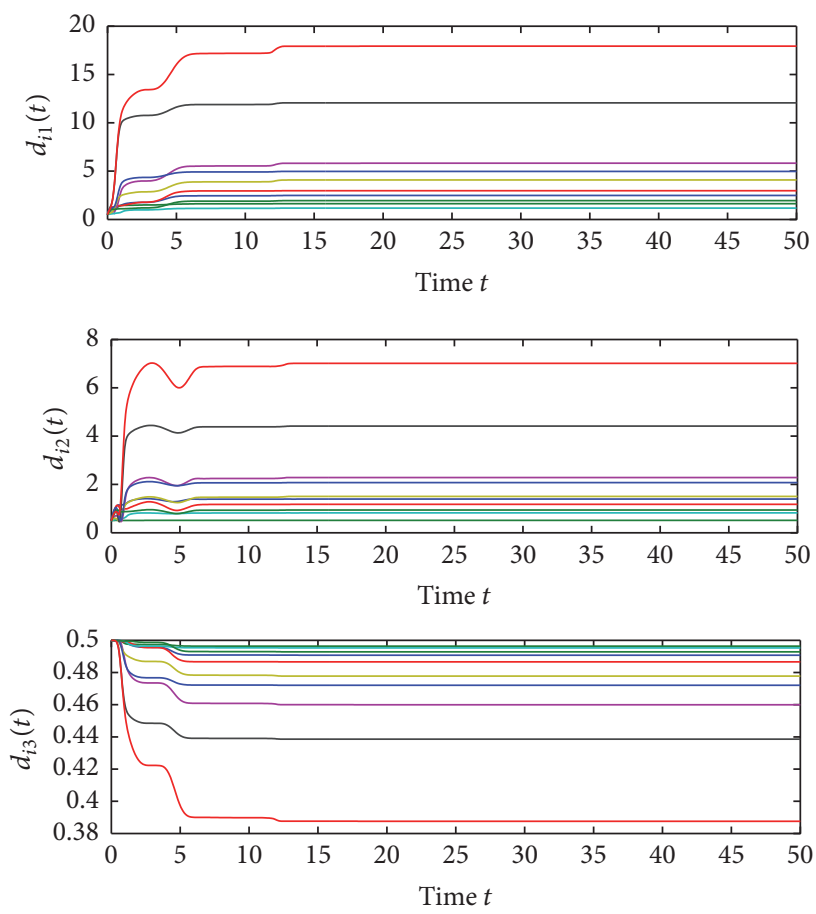

FIgURE 12: The evolution of adaptive pinning feedback gains $d_{i 1}(t)$, $d_{i 2}(t)$, and $d_{i 3}(t)(i=1,2, \ldots, 10)$.

\section{Conclusions}

In this paper, the hybrid adaptive pinning control for FPS of neural networks with mixed time-varying delays and uncertainties asymmetric coupling were investigated. We have applied the use of nonlinear and adaptive pinning controls and the nonlinear and adaptive controls. Some sufficient conditions are derived to guarantee the FPS by 

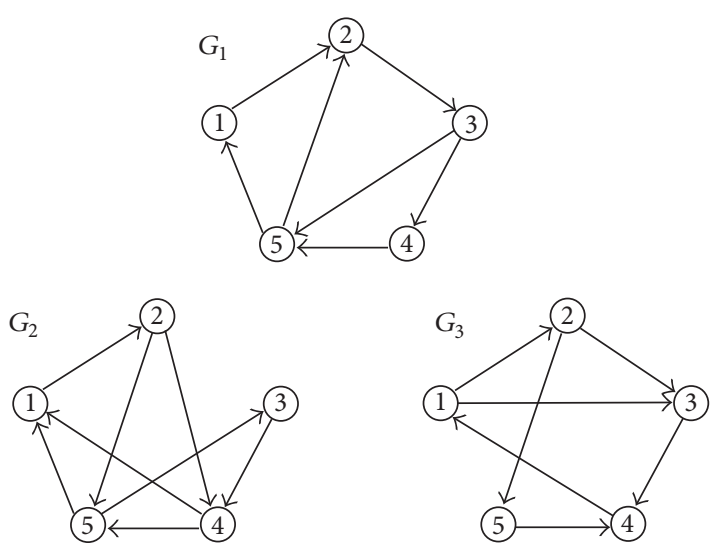

FIGURE 13: Simple directed neural network with 5 nodes.
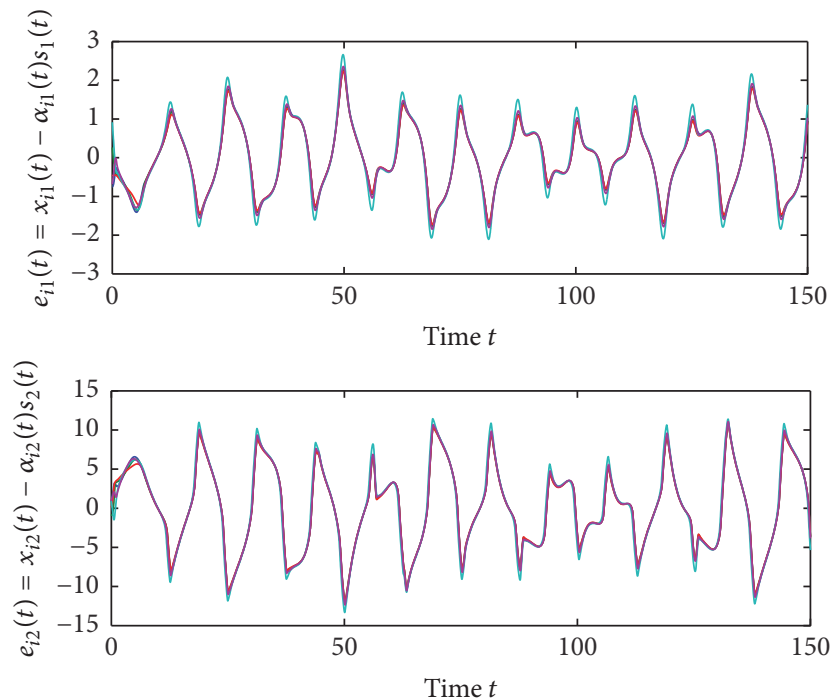

FIGURE 14: The FPS errors between the states of isolate node $\alpha(t) s(t)$ (45) and node $x_{i}(t)(47)$ without nonlinear and adaptive control (14).
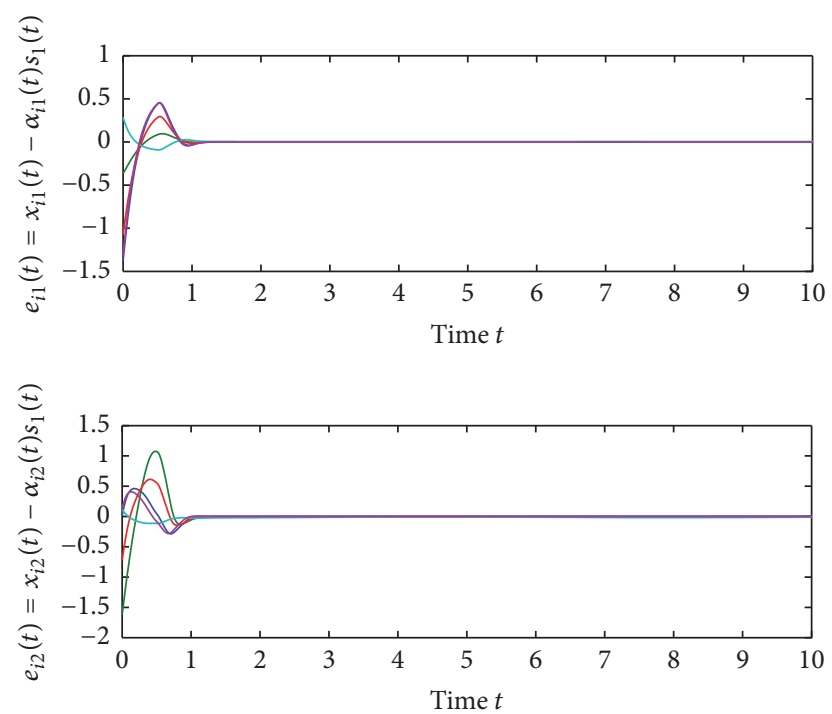

FIGURE 15: The FPS errors between the states of isolate node $\alpha(t) s(t)$ (45) and node $x_{i}(t)$ (47) with nonlinear and adaptive control (14).
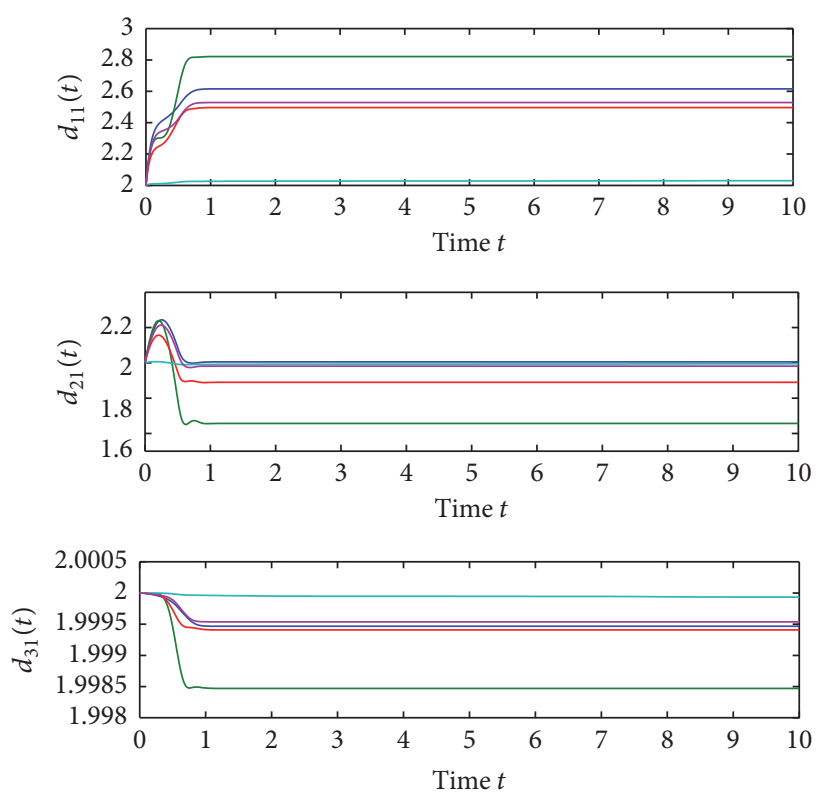

FIGURE 16: The evolution of adaptive feedback gains $d_{11}(t), d_{21}(t)$, and $d_{31}(t)$.

use of the Lyapunov-Krasovskii function method. Moreover, the drive and response systems could be synchronized up to the desired scaling functions based on the adaptive control technique. Furthermore, numerical examples are given to illustrate the effectiveness of the proposed theoretical results in this paper as well.

\section{Conflicts of Interest}

The authors declare that there are no conflicts of interest regarding the publication of this paper.

\section{Acknowledgments}

The first author was supported by National Research Council of Thailand and Khon Kaen University 2017 (Grant no. 600061). The second author was supported by Rajamangala University of Technology Isan and the Thailand Research Fund (TRF), the Office of the Higher Education Commission (OHEC) (Grant no. MRG5980027). The third author is also supported by Chiang Mai University, Chiang Mai, Thailand. The fourth author was financially supported by University of Phayao, Phayao, Thailand.

\section{References}

[1] A. Cichocki and R. Unbehauen, Neural networks for optimization and signal processing, Wiley, Hoboken, NJ, 1993.

[2] J. Wang and Z. Xu, "New study on neural networks: the essential order of approximation," Neural Networks, vol. 23, no. 5, pp. 618-624, 2010.

[3] J. Cao and J. Wang, "Global asymptotic stability of a general class of recurrent neural networks with time-varying delays," IEEE 
Transactions on Circuits and Systems I, vol. 50, no. 1, pp. 34-44, 2003.

[4] X.-M. Zhang and Q.-L. Han, "Global asymptotic stability analysis for delayed neural networks using a matrix-based quadratic convex approach," Neural Networks, vol. 54, pp. 57-69, 2014.

[5] K. Gu, V. L. Kharitonov, and J. Chen, Stability of Time-Delay System, Birkhauser, Boston, Mass, USA, 2003.

[6] Y. Zhang and Q.-L. Han, "Network-based synchronization of delayed neural networks," IEEE Transactions on Circuits and Systems I: Regular Papers, vol. 60, no. 3, pp. 676-689, 2013.

[7] K. Yuan, "Robust synchronization in arrays of coupled networks with delay and mixed coupling," Neurocomputing, vol. 72, no. 46, pp. 1026-1031, 2009.

[8] L. M. Pecora and T. L. Carroll, "Synchronization in chaotic systems," Physical Review Letters, vol. 64, no. 8, pp. 821-824, 1990.

[9] J. Cao, G. Chen, and P. Li, "Global synchronization in an array of delayed neural networks with hybrid coupling," IEEE Transactions on Systems, Man, and Cybernetics B: Cybernetics, vol. 38, no. 2, pp. 488-498, 2008.

[10] G. Chen, J. Zhou, and Z. Liu, "Global synchronization of coupled delayed neural networks and applications to chaotic CNN models," International Journal of Bifurcation and Chaos in Applied Sciences and Engineering, vol. 14, no. 7, pp. 2229-2240, 2004.

[11] M. J. Park, O. M. Kwon, J. H. Park, S. M. Lee, and E. J. Cha, "Synchronization criteria for coupled stochastic neural networks with time-varying delays and leakage delay," Journal of the Franklin Institute, vol. 349, no. 5, pp. 1699-1720, 2012.

[12] S. Chen and J. Cao, "Projective synchronization of neural networks with mixed time-varying delays and parameter mismatch," Nonlinear Dynamics, vol. 67, no. 2, pp. 1397-1406, 2012.

[13] H. Du, P. Shi, and N. Lü, "Function projective synchronization in complex dynamical networks with time delay via hybrid feedback control," Nonlinear Analysis: Real World Applications, vol. 14, no. 2, pp. 1182-1190, 2013.

[14] H. Du, "Function projective synchronization in complex dynamical networks with or without external disturbances via error feedback control," Neurocomputing, vol. 173, pp. 14431449, 2016.

[15] J. H. Hale, Theory of Functional Differential Equations, SpringerVerlag, New York, NY, USA, 1977.

[16] H. Dai, G. Si, and Y. Zhang, "Adaptive generalized function matrix projective lag synchronization of uncertain complex dynamical networks with different dimensions," Nonlinear Dynamics, vol. 74, no. 3, pp. 629-648, 2013.

[17] A. Abdurahman, H. Jiang, and Z. Teng, "Function projective synchronization of impulsive neural networks with mixed timevarying delays," Nonlinear Dynamics, vol. 78, no. 4, pp. 26272638, 2014.

[18] A. Abdurahman, H. Jiang, and K. Rahman, "Function projective synchronization of memristor-based Cohen-Grossberg neural networks with time-varying delays," Cognitive Neurodynamics, vol. 9, no. 6, pp. 603-613, 2015.

[19] X. Gao, G. Cai, and S. Cai, "Generalized function projective synchronization of weighted cellular neural networks with multiple time-varying coupling delays," in Proceedings of the 2013 6th International Conference on Biomedical Engineering and Informatics, BMEI 2013, pp. 760-764, china, December 2013.
[20] S. Zheng, Q. Bi, and G. Cai, "Adaptive projective synchronization in complex networks with time-varying coupling delay," Physics Letters A, vol. 373, no. 17, pp. 1553-1559, 2009.

[21] Y. Tang and W. K. Wong, "Distributed synchronization of coupled neural networks via randomly occurring control," IEEE Transactions on Neural Networks and Learning Systems, vol. 24, no. 3, pp. 435-447, 2013.

[22] J. Hu, J. Cao, A. Alofi, A. AL-Mazrooei, and A. Elaiw, "Pinning synchronization of coupled inertial delayed neural networks," Cognitive Neurodynamics, vol. 9, no. 3, pp. 341-350, 2015.

[23] J. Huang, C. Li, and Q. Han, "Stabilization of delayed chaotic neural networks by periodically intermittent control," Circuits, Systems, and Signal Processing, vol. 28, no. 4, pp. 567-579, 2009.

[24] S. Cai, X. Lei, and Z. Liu, "Outer synchronization between two hybrid-coupled delayed dynamical networks via aperiodically adaptive intermittent pinning control," Complexity, vol. 21, pp. 593-605, 2016.

[25] Q. Song and J. Cao, "On pinning synchronization of directed and undirected complex dynamical networks," IEEE Transactions on Circuits and Systems I: Regular Papers, vol. 57, no. 3, pp. 672-680, 2010.

[26] Q. Song, J. Cao, and F. Liu, "Pinning-controlled synchronization of hybrid-coupled complex dynamical networks with mixed time-delays," International Journal of Robust and Nonlinear Control, vol. 22, no. 6, pp. 690-706, 2012.

[27] D. Gong, F. L. Lewis, L. Wang, and K. Xu, "Synchronization for an array of neural networks with hybrid coupling by a novel pinning control strategy," Neural Networks, vol. 77, pp. 41-50, 2016.

[28] Q. Song, J. Cao, and F. Liu, "Pinning synchronization of linearly coupled delayed neural networks," Mathematics and Computers in Simulation, vol. 86, pp. 39-51, 2012.

[29] Q. Li, J. Guo, Y. Wu, and C.-Y. Sun, "Weighted Average Pinning Synchronization for a Class of Coupled Neural Networks with Time-Varying Delays," Neural Processing Letters, vol. 45, no. 1, pp. 95-108, 2017.

[30] A. R. Mitchell, "J. H. Wilkinson, The Algebraic Eigenvalue Problem (Clarendon Press, Oxford, 1965), 662pp., 110s., Proceedings of the Edinburgh Mathematical Society, vol. 15, no. 04, p. 328, 1967.

[31] D. J. Watts and S. H. Strogatz, "Collective dynamics of 'smallworld' networks," Nature, vol. 393, no. 6684, pp. 440-442, 1998.

[32] X. F. Wang and G. Chen, "Synchronization in scale-free dynamical networks: robustness and fragility," IEEE Transactions on Circuits and Systems I: Fundamental Theory and Applications, vol. 49, no. 1, pp. 54-62, 2002.

[33] R. Rakkiyappan, B. Kaviarasan, F. A. Rihan, and S. Lakshmanan, "Synchronization of singular Markovian jumping complex networks with additive time-varying delays via pinning control," Journal of the Franklin Institute, 2014.

[34] P. Niamsup, T. Botmart, and W. Weera, "Modified function projective synchronization of complex dynamical networks with mixed time-varying and asymmetric coupling delays via new hybrid pinning adaptive control," Advances in Difference Equations, vol. 2017, no. 1, 2017.

[35] T. Chen, X. Liu, and W. Lu, "Pinning complex networks by a single controller," IEEE Transactions on Circuits and Systems. I. Regular Papers, vol. 54, no. 6, pp. 1317-1326, 2007.

[36] X. F. Wang and G. Chen, "Pinning control of scale-free dynamical networks," Physica A, vol. 310, no. 3, pp. 521-531, 2002. 
[37] L. Shi, H. Zhu, S. Zhong, K. Shi, and J. Cheng, "Function projective synchronization of complex networks with asymmetric coupling via adaptive and pinning feedback control," ISA Transactions, vol. 65, pp. 81-87, 2016.

[38] M. MacDuffeec, The Theory of Matrices, Dover publications, New York, NY, USA, 2004.

[39] S. H. Strogatz, "Exploring complex networks," Nature, vol. 410, no. 6825 , pp. 268-276, 2001. 


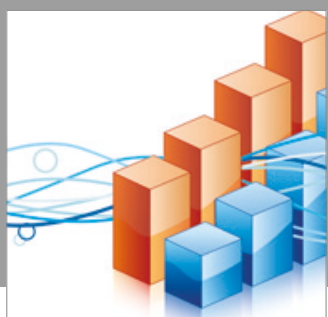

Advances in

Operations Research

vatersals

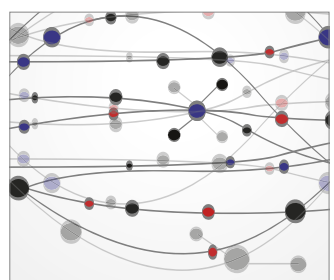

\section{The Scientific} World Journal
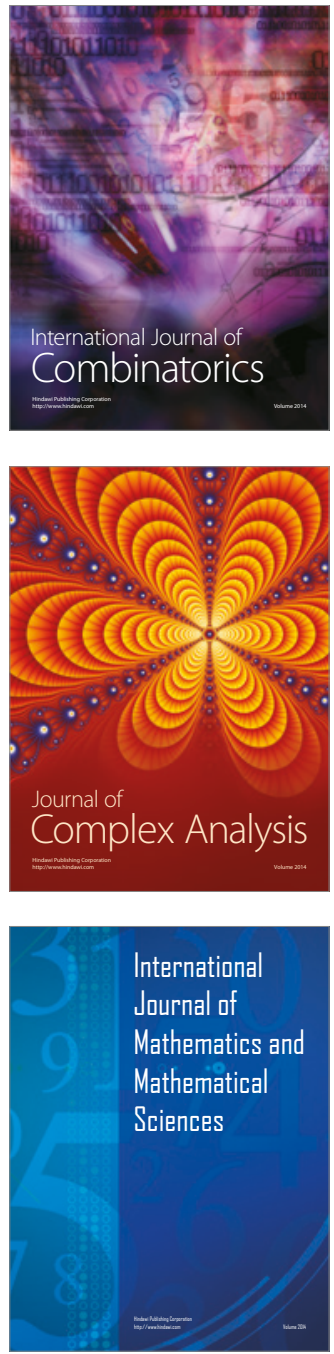
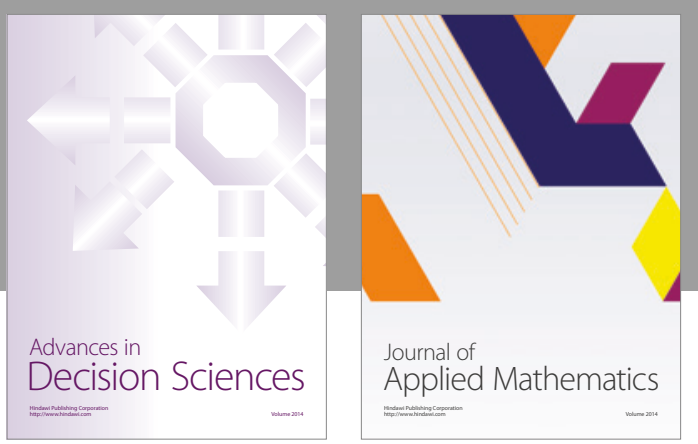

Algebra

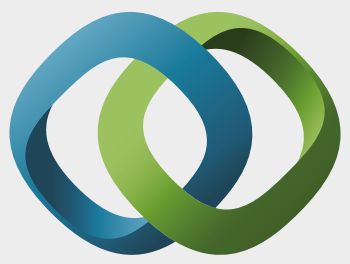

\section{Hindawi}

Submit your manuscripts at

https://www.hindawi.com
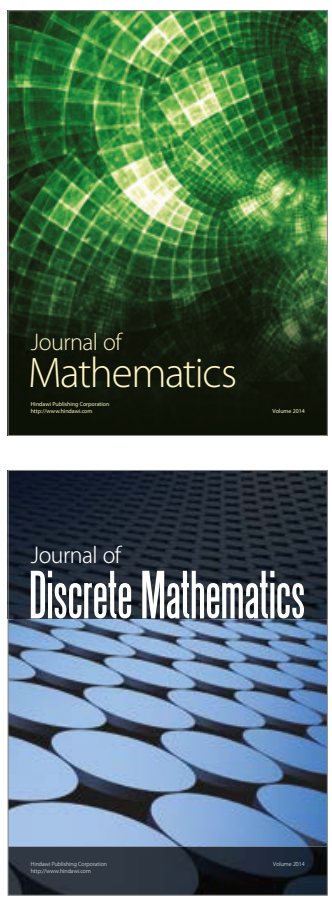

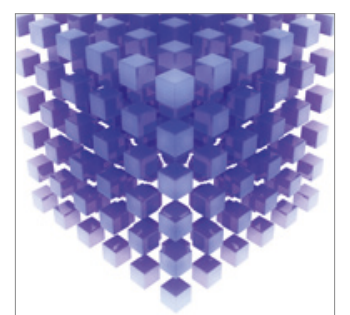

Mathematical Problems in Engineering
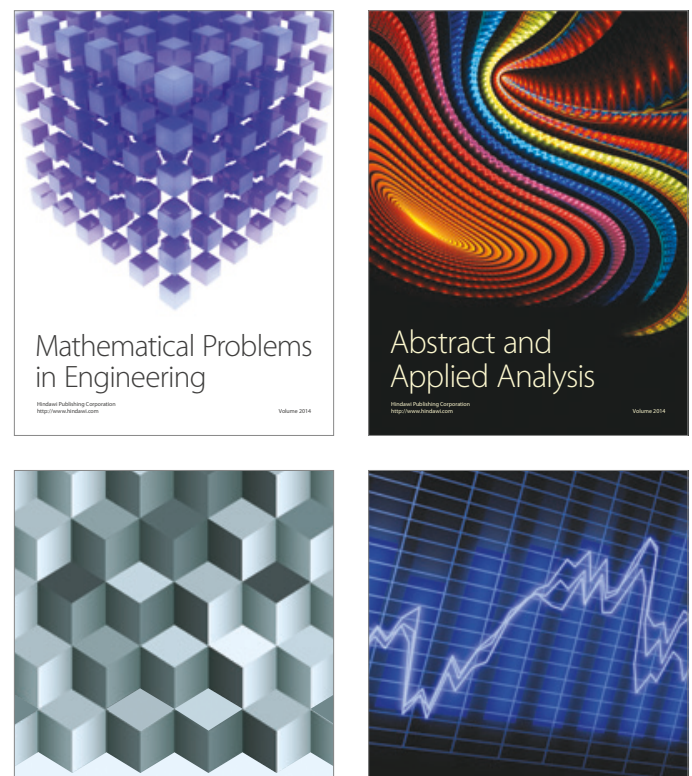

Journal of

Function Spaces

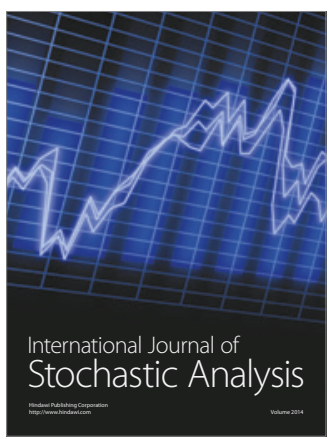

Probability and Statistics
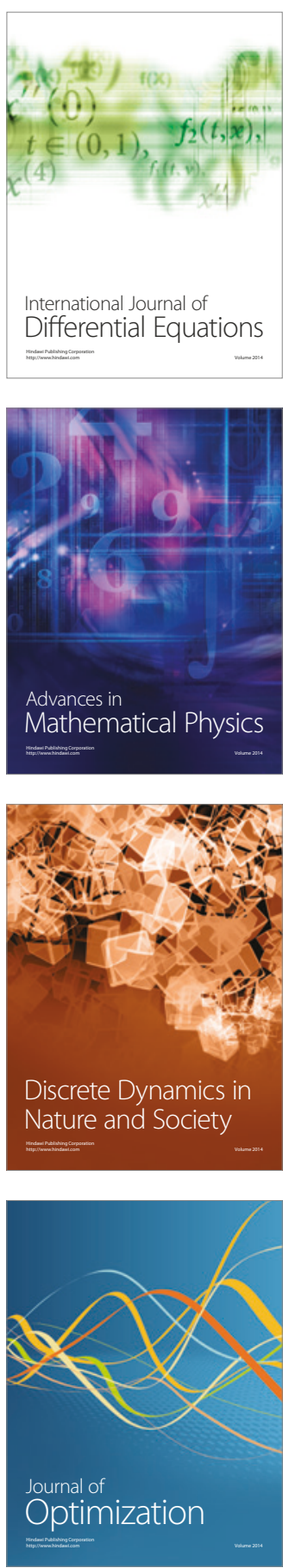